\title{
Integral and adelic aspects of the Mumford-Tate conjecture
}

\author{
by
}

\author{
Anna Cadoret and Ben Moonen
}

\begin{abstract}
Let $Y$ be an abelian variety over a subfield $k \subset \mathbb{C}$ that is of finite type over $\mathbb{Q}$. We prove that if the Mumford-Tate conjecture for $Y$ is true, then also some refined integral and adelic conjectures due to Serre are true for $Y$. In particular, if a certain Hodge-maximality condition is satisfied, we obtain an adelic open image theorem for the Galois representation on the (full) Tate module of $Y$. Our second main result is an (unconditional) adelic open image theorem for K3 surfaces. The proofs of these results rely on the study of a natural representation of the fundamental group of a Shimura variety.
\end{abstract}

AMS 2010 Mathematics Subject Classification: 11G18, 14G35

\section{Introduction}

This paper has two main results, one about abelian varieties, the other about K3 surfaces. The underlying mechanism is the same in both cases.

To explain the results, let $Y$ be either an abelian variety or a $\mathrm{K} 3$ surface over a subfield $k \subset \mathbb{C}$ that is finitely generated over $\mathbb{Q}$. Write $H=H_{1}(Y(\mathbb{C}), \mathbb{Z})$ in the first case and $H=H^{2}(Y(\mathbb{C}), \mathbb{Z})(1)$ if $Y$ is a K3 surface. Let $G_{\mathrm{B}} \subset \mathrm{GL}(H)$ be the Mumford-Tate group. We may identify $H \otimes \hat{\mathbb{Z}}$ with the étale cohomology of $Y$ with $\hat{\mathbb{Z}}$-coefficients $\left(H_{1}\right.$ or $\left.H^{2}(1)\right)$; this gives us a Galois representation

$$
\rho_{Y}: \operatorname{Gal}(\bar{k} / k) \rightarrow \operatorname{GL}(H)(\hat{\mathbb{Z}}) .
$$

It is known that, possibly after replacing $k$ with a finite extension, the image of $\rho_{Y}$ is contained in $G_{\mathrm{B}}(\hat{\mathbb{Z}})$. From now on, we assume this is the case. If $\ell$ is a prime number, let $\rho_{Y, \ell}: \operatorname{Gal}(\bar{k} / k) \rightarrow$ $\mathrm{GL}(H)\left(\mathbb{Z}_{\ell}\right)$ be the $\ell$-primary component of $\rho_{Y}$.

In the case of an abelian variety, our main result says that the usual Mumford-Tate conjecture implies an integral refinement of it. If moreover a certain maximality condition is satisfied (see Section 21), we obtain an adelic open image result for $\rho_{Y}$. These refined statements were conjectured by Serre. The precise result is as follows.

Theorem A. Let $Y$ be an abelian variety over $k$ for which the Mumford-Tate conjecture is true.

(i) The index $\left[G_{\mathrm{B}}\left(\mathbb{Z}_{\ell}\right): \operatorname{Im}\left(\rho_{Y, \ell}\right)\right]$ is bounded when $\ell$ varies. Moreover, for almost all $\ell$ the image of $\rho_{Y, \ell}$ contains the commutator subgroup of $G_{\mathrm{B}}\left(\mathbb{Z}_{\ell}\right)$, as well as the integral homotheties $\mathbb{Z}_{\ell}^{*} \cdot \mathrm{id}$.

(ii) If the Hodge structure $H_{1}(Y(\mathbb{C}), \mathbb{Q})$ is Hodge-maximal (see Def. 2.3), the image of $\rho_{Y}$ is an open subgroup of $G_{\mathrm{B}}\left(\mathbb{A}_{f}\right)$.

By a result of Larsen and Pink (see [11], Thm. 4.3), if the Mumford-Tate conjecture for an abelian variety is true for one prime number $\ell$, it is true for all $\ell$. The assumption that the Mumford-Tate conjecture for $Y$ is true is therefore unambiguous. Let us also note that Hodge-maximality is a necessary condition for the image of $\rho_{Y}$ to be open in $G_{\mathrm{B}}\left(\mathbb{A}_{f}\right)$; see Remark 2.6. 
In the case of a K3 surface, the Mumford-Tate conjecture is known (due to Tankeev [22] and, independently, André [1]), and we prove that the Hodge-maximality assumption is always satisfied. In this case we obtain the following adelic open image theorem.

Theorem B. If $Y$ is a $K 3$ surface, the image of $\rho_{Y}$ is an open subgroup of $G_{\mathrm{B}}\left(\mathbb{A}_{f}\right)$.

The proofs of these theorems rely on the fact that the moduli spaces of abelian varieties and K3 surfaces are (essentially) Shimura varieties.

In Section 3 we recall how to associate to a Shimura datum $(G, X)$ and a neat compact open subgroup $K_{0} \subset G\left(\mathbb{A}_{f}\right)$ a representation $\phi: \pi_{1}\left(S_{0}\right) \rightarrow K_{0} \subset G\left(\mathbb{A}_{f}\right)$, where $S_{0} \subset \operatorname{Sh}_{K_{0}}(G, X)$ (over some number field $F$ ) is a geometrically irreducible component. This reflects the fact that we have a tower of finite étale covers $\operatorname{Sh}_{K}(G, X) \rightarrow \operatorname{Sh}_{K_{0}}(G, X)$ indexed by the open subgroups $K \subset K_{0}$. For $\ell$ a prime number, let $\phi_{\ell}: \pi_{1}\left(S_{0}\right) \rightarrow K_{0, \ell}$ be the $\ell$-primary component of $\phi$.

The main technical result we prove, as Corollary 3.7, is that, under a mild assumption on the datum $(G, X)$, the image of the adelic representation $\phi$ is "big" in precisely the sense as in the conclusions of the main theorems. The proof of this result only involves abstract theory of Shimura varieties; it relies on Deligne's group-theoretic description of the reciprocity law that gives the Galois action on the geometric connected components of $\operatorname{Sh}(G, X)$.

To understand how this result leads to our main theorems, we have to link the representation $\phi$ to the Galois representation $\rho_{Y}$ on the cohomology of $Y$. Taking $G$ to be the Mumford-Tate group of $Y$, we obtain a Shimura variety that has an interpretation as a moduli space of abelian varieties or K3 surfaces with additional structures. If $y \in S_{0}(k)$ is the point corresponding to $Y$, we obtain a homomorphism $\sigma_{y}: \pi_{1}(y) \rightarrow \pi_{1}\left(S_{0}\right)$ (with $\pi_{1}(y) \cong \operatorname{Gal}(\bar{k} / k)$ ), and the composition $\phi \circ \sigma_{y}$ is isomorphic to the Galois representation $\rho_{Y}$. Using a classical result of Bogomolov, the assumption that the Mumford-Tate conjecture for $Y$ is true, for some prime number $\ell$, implies that the image of $\phi_{\ell} \circ \sigma_{y}$ is open in the image of $\phi_{\ell}$. Points $y \in S_{0}(k)$ for which this holds are said to be $\ell$-Galois-generic with respect to $\phi$. For points on a Shimura variety of abelian type, a result of the first author and Kret says that being $\ell$-Galois-generic for some $\ell$ implies something that is a priori much stronger, namely that the image of the adelic representation $\phi \circ \sigma_{y}$ is open in the image of $\phi$. (See [6], Thm. 1.1. This result is a consequence of the open adelic image theorem for abelian schemes proven by the first author in [5].) Our main results are obtained by combining this with our result on the image of $\phi$.

The first two sections of the paper are of a preliminary nature. We recall some conjectures due to Serre that refine the Mumford-Tate conjecture. Also we discuss the notion of (Hodge-)maximality, which is a necessary condition for an adelic open image theorem to hold. Section 3 forms the core of the paper. We define the representation $\phi$ associated with a Shimura variety; further we state and prove the main result, Theorem 3.6 and its Corollary 3.7 about the image of $\phi$. In Section 4 we briefly recall various notions of Galois-genericity, and we state the result of Cadoret-Kret that we need. In Section 5, which is devoted to abelian varieties, we prove Theorem A. Also we give examples of abelian varieties for which the $H_{1}$ is not Hodge-maximal. These examples suggest that Hodge-maximality depends in a rather subtle way on the structure of the Mumford-Tate group. Finally, in Section 6 we discuss K3 surfaces. We prove that the $H^{2}(1)$ of a $\mathrm{K} 3$ surface is always Hodge-maximal, and we deduce Theorem B.

Acknowledgement. We thank Akio Tamagawa for his interest and for helpful discussions. 


\section{An integral variant of the Mumford-Tate conjecture}

1.1 Let $Y$ be a smooth proper scheme of finite type over a subfield $k$ of $\mathbb{C}$ that is finitely generated over $\mathbb{Q}$. Fix integers $i$ and $n$.

Let $H=H^{i}(Y(\mathbb{C}), \mathbb{Z})(n) /($ torsion $)$, which is a polarizable Hodge structure of weight $i-2 n$. We denote by $G_{\mathrm{B}} \subset \mathrm{GL}(H)$ the Mumford-Tate group. By this we mean that the generic fibre $G_{\mathrm{B}, \mathbb{Q}} \subset$ $\mathrm{GL}\left(H_{\mathbb{Q}}\right)$ is the Mumford-Tate group of $H_{\mathbb{Q}}$ in the usual sense, and that $G_{\mathrm{B}}$ is the Zariski closure of $G_{\mathrm{B}, \mathbb{Q}}$ inside $\mathrm{GL}(H)$. If the context requires it, we include $Y$ in the notation, writing $G_{\mathrm{B}, Y}$, etc.

For a prime number $\ell$, let $H_{\ell}=H^{i}\left(Y_{\bar{k}}, \mathbb{Z}_{\ell}\right)(n) /\left(\right.$ torsion), which is a free $\mathbb{Z}_{\ell}$-module of finite rank on which we have a continuous Galois representation

$$
\rho_{\ell}: \operatorname{Gal}(\bar{k} / k) \rightarrow \mathrm{GL}\left(H_{\ell}\right) .
$$

We denote by $G_{\ell} \subset \mathrm{GL}\left(H_{\ell}\right)$ the Zariski closure of the image of $\rho_{\ell}$. The generic fibre $G_{\ell, \mathbb{Q}_{\ell}} \subset \mathrm{GL}\left(H_{\ell, \mathbb{Q}_{\ell}}\right)$ is the Zariski closure of the image of the Galois representation on $H_{\ell, \mathbb{Q}_{\ell}}$. We define $G_{\ell}^{0} \subset G_{\ell}$ to be the Zariski closure of the identity component $\left(G_{\ell, \mathbb{Q}_{\ell}}\right)^{0}$.

If we replace $k$ by a finitely generated extension, $G_{\ell}$ may become smaller, but its identity component $G_{\ell}^{0}$ does not change. By a result of Serre (see [20] or [10], Prop. 6.14), there exists a finite field extension $k \subset k^{\text {conn }}$ in $\mathbb{C}$ (depending on $Y, i$ and $n$ ) such that for every field $K$ that contains $k^{\text {conn }}$ and every prime number $\ell$, the generic fibre of $G_{\ell, Y_{K}}$ is connected.

Via the comparison isomorphism $H \otimes \mathbb{Z}_{\ell} \stackrel{\sim}{\rightarrow} H_{\ell}$, we may view $G_{\mathrm{B}} \otimes \mathbb{Z}_{\ell}$ as a subgroup scheme of $\mathrm{GL}\left(H_{\ell}\right)$.

1.2 Mumford-Tate Conjecture. With notation as above, $G_{\mathrm{B}} \otimes \mathbb{Z}_{\ell}=G_{\ell}^{0}$ as subgroup schemes of $\operatorname{GL}\left(H_{\ell}\right)$.

Note that, though we have stated the conjecture using group schemes over $\mathbb{Z}_{\ell}$, the Mumford-Tate conjecture in this form is equivalent to the conjecture that $G_{\mathrm{B}} \otimes \mathbb{Q}_{\ell}$ equals $G_{\ell, \mathbb{Q}_{\ell}}^{0}$ as algebraic subgroups of $\operatorname{GL}\left(H_{\ell, \mathbb{Q}_{\ell}}\right)$, which is the Mumford-Tate conjecture as it is usually stated. As $H_{\ell}$ is Hodge-Tate, it follows from a result of Bogomolov [2] (with some extensions due to Serre; see also [20]) that the image of $\rho_{\ell}$ is open in $G_{\ell}\left(\mathbb{Q}_{\ell}\right)$. Hence the Mumford-Tate conjecture is equivalent to the assertion that $\operatorname{Im}\left(\rho_{\ell}\right)$ is an open subgroup of $G_{\mathrm{B}}\left(\mathbb{Z}_{\ell}\right)$ (assuming $k=k^{\text {conn }}$ ). Further note that the Mumford-Tate conjecture depends, a priori, on $\ell$ and also on the chosen complex embedding of $k$.

The following strengthening of the Mumford-Tate conjecture was proposed by Serre; see Conjecture C.3.7 in [19] and cf. [21].

1.3 Integral Mumford-Tate Conjecture (Serre). Retain the notation of 1.1, and assume $k=$ $k^{\mathrm{conn}}$. Then for all $\ell$ the image $\operatorname{Im}\left(\rho_{\ell}\right)$ is contained in $G_{\mathrm{B}}\left(\mathbb{Z}_{\ell}\right)$ as an open subgroup, and the index $\left[G_{\mathrm{B}}\left(\mathbb{Z}_{\ell}\right): \operatorname{Im}\left(\rho_{\ell}\right)\right]$ is bounded when $\ell$ varies. Further, for almost all $\ell$ the image of $\rho_{\ell}$ contains the commutator subgroup of $G_{\mathrm{B}}\left(\mathbb{Z}_{\ell}\right)$ and all homotheties of the form $c^{i-2 n} \cdot \mathrm{id}$, for $c \in \mathbb{Z}_{\ell}^{*}$.

Compared with the usual Mumford-Tate conjecture, the main point in the above conjecture is that it should be possible to bound the index of $\operatorname{Im}\left(\rho_{\ell}\right)$ in $G_{\mathrm{B}}\left(\mathbb{Z}_{\ell}\right)$ by a constant independent of $\ell$. 


\section{Maximality, and an adelic form of the Mumford-Tate conjecture}

2.1 Definition. Let $M$ be a connected algebraic group over a field $k$ of characteristic 0 . Let $k \subset F$ be a field extension, $S$ an algebraic group over $F$, and $h: S \rightarrow M_{F}$ a homomorphism. Then we say that $h$ is maximal if there is no non-trivial isogeny of connected $k$-groups $M^{\prime} \rightarrow M$ such that $h$ lifts to a homomorphism $S \rightarrow M_{F}^{\prime}$.

Note that if $F$ is algebraically closed, maximality of $h$ only depends on its $M(F)$-conjugacy class.

2.2 The following remarks closely follow [27, 0.2. Let $M$ be a connected reductive group over a subfield $k \subset \mathbb{C}$. Let $\mathscr{C}$ be a conjugacy class of complex cocharacters $\mu: \mathbb{G}_{\mathrm{m}, \mathbb{C}} \rightarrow M_{\mathbb{C}}$. Let $\pi_{1}(M)$ denote the fundamental group of $M$ as defined by Borovoi in $[3]$. This is a finitely generated $\mathbb{Z}$ module with a continuous action of $\Gamma=\operatorname{Gal}(\bar{k} / k)$. If $\left(X^{*}, R, X_{*}, R^{\vee}\right)$ is the root datum of $M_{\bar{k}}$ and $Q\left(R^{\vee}\right)=\left\langle R^{\vee}\right\rangle \subset X_{*}$ is the coroot lattice, $\pi_{1}(M) \cong X_{*} / Q\left(R^{\vee}\right)$.

The conjugacy class $\mathscr{C}$ of complex cocharacters corresponds to an orbit $\mathscr{C} \subset X_{*}$ under the Weyl group $W$. As the induced $W$-action on $\pi_{1}(M)$ is trivial, any two elements in $\mathscr{C}$ have the same image in $\pi_{1}(M)$; call it $[\mathscr{C}] \in \pi_{1}(M)$.

If $M^{\prime}$ is a connected reductive $k$-group and $f: M^{\prime} \rightarrow M$ is an isogeny, the map induced by $f$ identifies $\pi_{1}\left(M^{\prime}\right)$ with a $\mathbb{Z}[\Gamma]$-submodule of finite index in $\pi_{1}(M)$. Conversely, every such submodule comes from an isogeny of connected $k$-groups, which is unique up to isomorphism over $M$. A conjugacy class $\mathscr{C}$ as above lifts to $M^{\prime}$ if and only if $[\mathscr{C}] \in \pi_{1}\left(M^{\prime}\right)$.

We shall usually be in a situation where the $\mathbb{Z}[\Gamma]$-submodule spanned by $[\mathscr{C}]$ has finite index in $\pi_{1}(M)$. (See below.) In this case, there is a uniquely determined maximal isogeny $M^{\prime} \rightarrow M$ of connected $k$-groups such that the $\mu \in \mathscr{C}$ lift to complex cocharacters of $M^{\prime}$. Further, the cocharacters $\mu \in \mathscr{C}$ are maximal in the sense of Definition 2.1] if and only if $[\mathscr{C}]$ generates $\pi_{1}(M)$ as a $\mathbb{Z}[\Gamma]$-module.

2.3 Definition. Let $V$ be a $\mathbb{Q}$-Hodge structure, given by the homomorphism $h: \mathbb{S} \rightarrow \operatorname{GL}(V)_{\mathbb{R}}$. Let $M \subset \operatorname{GL}(V)$ be the Mumford-Tate group. Then $V$ is said to be Hodge-maximal if $h: \mathbb{S} \rightarrow M_{\mathbb{R}}$ is maximal in the sense of Definition 2.1.

Hodge-maximality of $V$ is equivalent to the condition that the associated cocharacter $\mu: \mathbb{G}_{\mathrm{m}, \mathbb{C}} \rightarrow$ $M_{\mathbb{C}}$ is maximal. This allows us to apply 2.2, taking $k=\mathbb{Q}$ and $\Gamma=\operatorname{Gal}(\overline{\mathbb{Q}} / \mathbb{Q})$. If $\mathscr{C}$ is the $M(\mathbb{C})$ conjugacy class of $\mu$, the assumption that $M$ is the Mumford-Tate group of $V$ implies that the $\mathbb{Z}[\Gamma]$ submodule of $X_{*}(M)$ generated by $\mathscr{C}$ has finite index. Hence also the $\mathbb{Z}[\Gamma]$-submodule of $\pi_{1}(M)$ generated by $[\mathscr{C}]$ has finite index. By what was explained in $2.2, V$ is Hodge-maximal if and only if $\mathbb{Z}[\Gamma] \cdot[\mathscr{C}]=\pi_{1}(M)$.

2.4 Retaining the notation and assumptions of 1.1, let $\hat{H}=\prod_{\ell} H_{\ell}$, where the product is taken over all prime numbers $\ell$. We then have a continuous Galois representation

$$
\rho: \operatorname{Gal}(\bar{k} / k) \rightarrow \operatorname{GL}(\hat{H})
$$

whose $\ell$-primary component is the representation $\rho_{\ell}$ defined in 1.1. The comparison isomorphism between singular and étale cohomology gives an isomorphism $\hat{H} \cong H \otimes \hat{\mathbb{Z}}$; via this we may view $\rho$ as a representation taking values in $\operatorname{GL}(H)(\hat{\mathbb{Z}})$.

The following adelic version of the Mumford-Tate conjecture was proposed by Serre; see Conjecture C.3.8 in [19. 
2.5 Adelic Mumford-Tate Conjecture (Serre). With notation as in 1.1, suppose that $k=k^{\text {conn }}$ and that the Hodge structure $H$ is Hodge-maximal. Then $\operatorname{Im}(\rho)$ is an open subgroup of $G_{\mathrm{B}}\left(\mathbb{A}_{f}\right)$.

2.6 Remark. It follows from a result of Wintenberger that the Hodge-maximality of $H$ is essential. (For simplicity we shall assume here that the ground field $k$ is a number field.) Indeed, suppose there exists a non-trivial isogeny of $\mathbb{Q}$-groups $M^{\prime} \rightarrow G_{\mathrm{B}}$ with $M^{\prime}$ connected, such that $h: \mathbb{S} \rightarrow G_{\mathrm{B}, \mathbb{R}}$ lifts to a homomorphism $\mathbb{S} \rightarrow M_{\mathbb{R}}^{\prime}$. By [26], Théorème 2.1.7, and possibly after replacing the ground field $k$ with a finite extension, the $\ell$-adic representations $\rho_{\ell}$ lift to Galois representations with values in $M^{\prime}$. On the other hand, it follows from [15], Proposition 6.4, that the image of $M^{\prime}\left(\mathbb{A}_{f}\right) \rightarrow G_{\mathrm{B}}\left(\mathbb{A}_{f}\right)$ is not open in $G_{\mathrm{B}}\left(\mathbb{A}_{f}\right)$; hence $\operatorname{Im}(\rho)$ cannot be open in $G_{\mathrm{B}}\left(\mathbb{A}_{f}\right)$.

2.7 Let $(G, X)$ be a Shimura datum such that $G$ is the generic Mumford-Tate group on $X$. By definition, this means that there exist points $h \in X$ for which there is no proper subgroup $G^{\prime} \subset G$ such that $h: \mathbb{S} \rightarrow G_{\mathbb{R}}$ factors through $G_{\mathbb{R}}^{\prime}$. The locus of points $h$ for which this holds forms a subset $X^{\text {Hgen }} \subset X$ called the Hodge-generic locus.

Similar to the definition in 2.3, we say that $(G, X)$ is maximal if there is no non-trivial isogeny of Shimura data $f:\left(G^{\prime}, X^{\prime}\right) \rightarrow(G, X)$. (Note that $G^{\prime}$ is necessarily connected, as it is part of a Shimura datum.) Clearly, if $(G, X)$ is maximal then all $h: \mathbb{S} \rightarrow G_{\mathbb{R}}$ in $X^{\text {Hgen }}$ are maximal in the sense of Definition 2.1. Conversely, if some $h \in X^{\text {Hgen }}$ is maximal then $(G, X)$ is maximal. (If we have $f$ as above, $f\left(X^{\prime}\right) \subset X$ is a union of connected components but need not be the whole $X$; however, changing $f$ by an inner automorphism of $G$ we can always ensure that some given $h \in X$ lies in $f\left(X^{\prime}\right)$.)

To each $h \in X$ corresponds a complex cocharacter $\mu_{h}$ of $G$, and the $\mu_{h}$ thus obtained all lie in a single $G(\mathbb{C})$-conjugacy class $\mathscr{C}(G, X)$. It follows from the previous remarks that $(G, X)$ is maximal if and only if the associated class $[\mathscr{C}(G, X)]$ generates $\pi_{1}(G)$ as a $\mathbb{Z}[\Gamma]$-module.

2.8 Remarks. (i) Let $f:\left(G_{1}, X_{1}\right) \rightarrow\left(G_{2}, X_{2}\right)$ be a morphism of Shimura data with $f: G_{1} \rightarrow G_{2}$ surjective. If $G_{1}$ is the generic Mumford-Tate group on $X_{1}$ then $G_{2}$ is the generic Mumford-Tate group on $X_{2}$. If $f: G_{1} \rightarrow G_{2}$ is an isogeny then also the converse is true.

(ii) If in (i) $\operatorname{Ker}(f)$ is semisimple then also maximality is preserved: if $\left(G_{1}, X_{1}\right)$ is maximal, so is $\left(G_{2}, X_{2}\right)$. Indeed, in this case $\pi_{1}\left(G_{2}\right)$ is a quotient of $\pi_{1}\left(G_{1}\right)$ in such a way that $\left[\mathscr{C}\left(G_{2}, X_{2}\right)\right]$ is the image of $\left[\mathscr{C}\left(G_{1}, X_{1}\right)\right]$.

(iii) Given a Shimura datum $(G, X)$, it follows from the remarks in 2.2 that, up to isomorphism, there exists a unique isogeny of Shimura data $f:(\tilde{G}, \tilde{X}) \rightarrow(G, X)$ such that $(\tilde{G}, \tilde{X})$ is maximal. By (i), $G$ is the generic Mumford-Tate group on $X$ if and only if $\tilde{G}$ is the generic Mumford-Tate group on $\tilde{X}$.

\section{Adelic representations associated with Shimura varieties}

3.1 Let $(G, X)$ be a Shimura datum. Throughout we assume that $G$ is the generic Mumford-Tate group on $X$. (See 2.7) In this case, conditions $(2.1 .1 .1-5)$ of [8] are satisfied and $Z(\mathbb{Q})$ is discrete in $Z\left(\mathbb{A}_{f}\right)$. (Cf. [8], 2.1.11; for details see also [24], Lemma 5.13.)

If $K \subset G\left(\mathbb{A}_{f}\right)$ is a compact open subgroup, we have $\operatorname{Sh}_{K}(G, X)(\mathbb{C})=G(\mathbb{Q}) \backslash X \times G\left(\mathbb{A}_{f}\right) / K$. For $h \in X$ and $\gamma K \in G\left(\mathbb{A}_{f}\right) / K$, let $[h, \gamma K]$ denote the corresponding $\mathbb{C}$-valued point of $\operatorname{Sh}_{K}(G, X)$.

Let $K_{0} \subset G\left(\mathbb{A}_{f}\right)$ be a neat compact open subgroup. If $K \subset K_{0}$ is an open subgroup, the induced morphism on Shimura varieties $\operatorname{Sh}_{K, K_{0}}: \mathrm{Sh}_{K}(G, X) \rightarrow \mathrm{Sh}_{K_{0}}(G, X)$ is finite étale. If, moreover, $K$ is normal in $K_{0}$, this morphism is Galois with group $K_{0} / K$. 
Choose a point $h_{0} \in X$. Let $S_{0, \mathbb{C}}$ be the irreducible component of $\operatorname{Sh}_{K_{0}}(G, X)_{\mathbb{C}}$ that contains the point $\left[h_{0}, e K_{0}\right]$. Let $F$ be the field of definition of this component, which is a finite extension of the reflex field $E(G, X)$. To simplify notation, we write $\operatorname{Sh}_{K}$ for $\operatorname{Sh}_{K}(G, X)_{F}$. By construction, we have a geometrically irreducible component $S_{0} \subset \mathrm{Sh}_{K_{0}}$.

For $K$ an open normal subgroup of $K_{0}$, let $S_{K} \subset \mathrm{Sh}_{K}$ be the inverse image of $S_{0} \subset \mathrm{Sh}_{K_{0}}$ under the transition morphism $\mathrm{Sh}_{K, K_{0}}$. Then $S_{K} \rightarrow S_{0}$ is étale Galois with group $K_{0} / K$.

Let $\bar{s}_{K}=\left[h_{0}, e K\right] \in S_{K}(\mathbb{C})$. The system of points $\bar{s}=\left(\bar{s}_{K}\right)$ thus obtained is compatible in the sense that $\operatorname{Sh}_{K_{2}, K_{1}}\left(\bar{s}_{K_{2}}\right)=\bar{s}_{K_{1}}$ for $K_{2} \subset K_{1} \subset K_{0}$. We abbreviate $\bar{s}_{K_{0}}$ to $\bar{s}_{0}$. With this choice of base points, $S_{K} \rightarrow S_{0}$ corresponds to a homomorphism $\phi_{K}: \pi_{1}\left(S_{0}, \bar{s}_{0}\right) \rightarrow K_{0} / K$. If $K_{2} \subset K_{1}$ are open normal subgroups of $K_{0}$, the homomorphism $\phi_{K_{1}}$ equals the composition of $\phi_{K_{2}}: \pi_{1}\left(S_{0}, \bar{s}_{0}\right) \rightarrow K_{0} / K_{2}$ and the canonical map $K_{0} / K_{2} \rightarrow K_{0} / K_{1}$. We may therefore pass to the limit; as the intersection of all open normal subgroups $K \subset K_{0}$ is trivial, this gives a continuous homomorphism

$$
\phi_{\bar{s}}: \pi_{1}\left(S_{0}, \bar{s}_{0}\right) \rightarrow K_{0} .
$$

3.2 Remarks. (i) The homomorphism $\phi$ is functorial in the following sense. Let $f:(G, X) \rightarrow$ $\left(G^{\prime}, X^{\prime}\right)$ be a morphism of Shimura data. On reflex fields we have $E\left(G^{\prime}, X^{\prime}\right) \subset E=E(G, X)$. Let $K_{0} \subset$ $G\left(\mathbb{A}_{f}\right)$ and $K_{0}^{\prime} \subset G^{\prime}\left(\mathbb{A}_{f}\right)$ be neat compact open subgroups with $f\left(K_{0}\right) \subset K_{0}^{\prime}$. Choose $h_{0} \in X$ and let $h_{0}^{\prime}=f\left(h_{0}\right) \in X^{\prime}$. As in 3.1, this gives rise to geometrically irreducible components $S_{0} \subset \operatorname{Sh}_{K_{0}}(G, X)_{F}$ and $S_{0}^{\prime} \subset \operatorname{Sh}_{K_{0}^{\prime}}\left(G^{\prime}, X^{\prime}\right)_{F^{\prime}}$, and it is easy to see that $E F^{\prime} \subset F$. Further, $h_{0}$ and $h_{0}^{\prime}$ give rise to compatible systems of base points $\bar{s}=\left(\bar{s}_{K}\right)$ and $\bar{s}^{\prime}=\left(\bar{s}_{K^{\prime}}^{\prime}\right)$. The morphism $\operatorname{Sh}(f): \operatorname{Sh}_{K_{0}}(G, X) \rightarrow \operatorname{Sh}_{K_{0}^{\prime}}\left(G^{\prime}, X^{\prime}\right)_{E}$ restricts to a morphism $S_{0} \rightarrow S_{0, F}^{\prime}$ over $F$ with $\bar{s}_{0} \mapsto \bar{s}_{0}^{\prime}$. We then have a commutative diagram

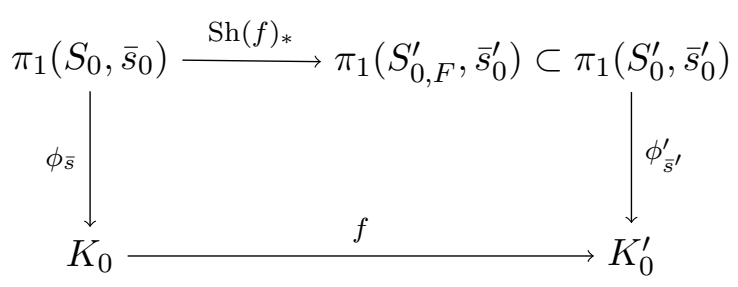

(ii) The homomorphism $\phi$ is essentially independent of the choice of $h_{0} \in X$ and the resulting system of base points $\bar{s}$. If we choose another point $h_{0}^{\prime} \in X$ that lies in the same connected component as $h_{0}$, this gives rise to a different collection of base points $\bar{s}^{\prime}$. There is a canonically determined conjugacy class of isomorphisms $\alpha: \pi_{1}\left(S_{0}, \bar{s}_{0}\right) \stackrel{\sim}{\rightarrow} \pi_{1}\left(S_{0}, \bar{s}_{0}^{\prime}\right)$. For $\alpha$ in this class, the homomorphisms $\phi_{\bar{s}}$ and $\phi_{\bar{s}^{\prime}} \circ \alpha$ differ by an inner automorphism of $K_{0}$.

If $h_{0}^{\prime}$ lies in a different connected component of $X$, there exists an inner automorphism $\alpha=\operatorname{Inn}(g)$ of $(G, X)$ such that $\alpha\left(h_{0}\right)$ and $h_{0}^{\prime}$ lie in the same component of $X$. By functoriality together with the previous case, it follows that the associated representations $\phi_{\bar{s}}$ and $\phi_{\bar{s}^{\prime}}$ are conjugate when we restrict to suitable subgroups of finite index in the respective $\pi_{1}$ 's.

In view of the above remarks, we shall from now on omit the base point $\bar{s}_{0}$ from the notation, unless it plays a role in the discussion.

3.3 Our main goal in this section is to describe the image of the representation $\phi=\phi_{\bar{s}}$ defined in 3.1 , In order to do this, we need to recall some definitions and results from the theory of Shimura varieties. For proofs of the stated results we refer to [8], Section 2. Throughout, $(G, X)$ is a Shimura datum as in 3.1. Let $G(\mathbb{R})_{+} \subset G(\mathbb{R})$ be the subgroup of elements that are mapped into the identity component $G^{\text {ad }}(\mathbb{R})^{+} \subset G^{\text {ad }}(\mathbb{R})$ (for the Euclidean topology) under the adjoint map. 
Let $\gamma: \tilde{G} \rightarrow G^{\text {der }}$ denote the simply connected cover of the derived group of $G$. Then $G(\mathbb{Q}) \gamma \tilde{G}(\mathbb{A})$ is a closed normal subgroup of $G(\mathbb{A})$, and we define $\pi(G)=G(\mathbb{A}) / G(\mathbb{Q}) \gamma \tilde{G}(\mathbb{A})$. Next define $\bar{\pi}_{0} \pi(G)=$ $\pi_{0} \pi(G) / \pi_{0} G(\mathbb{R})_{+}$, where $\pi_{0}$ means the group of connected components. This $\bar{\pi}_{0} \pi(G)$ is an abelian profinite group.

Define $G(\mathbb{Q})_{+}=G(\mathbb{Q}) \cap G(\mathbb{R})_{+}$, and let $G(\mathbb{Q})_{+}^{-}$denote its closure inside $G\left(\mathbb{A}_{f}\right)$. The natural homomorphism $G\left(\mathbb{A}_{f}\right) \rightarrow \bar{\pi}_{0} \pi(G)$ induces an isomorphism $G\left(\mathbb{A}_{f}\right) / G(\mathbb{Q})_{+}^{-} \stackrel{\sim}{\rightarrow} \bar{\pi}_{0} \pi(G)$. If there is no risk of confusion we identify the two groups.

The group $G\left(\mathbb{A}_{f}\right)$ acts on the Shimura variety $\operatorname{Sh}(G, X)$ from the right. This action makes the set $\pi_{0}\left(\operatorname{Sh}(G, X)_{\mathbb{C}}\right)$ a torsor under $\bar{\pi}_{0} \pi(G)$.

3.4 Let $E=E(G, X)$ be the reflex field and $E^{\mathrm{ab}}$ its maximal abelian extension. As $\pi_{0}\left(\operatorname{Sh}(G, X)_{\mathbb{C}}\right)$ is a torsor under $\bar{\pi}_{0} \pi(G)$, which is abelian, the action of $\operatorname{Gal}(\bar{E} / E)$ on $\pi_{0}\left(\operatorname{Sh}(G, X)_{\mathbb{C}}\right)$ gives rise to a well-determined homomorphism

$$
\text { rec: } \operatorname{Gal}\left(E^{\mathrm{ab}} / E\right) \rightarrow \bar{\pi}_{0} \pi(G) \cong G\left(\mathbb{A}_{f}\right) / G(\mathbb{Q})_{+}^{-},
$$

called the reciprocity homomorphism.

Let $q: G\left(\mathbb{A}_{f}\right) \rightarrow \bar{\pi}_{0} \pi(G)$ be the canonical map. For $K \subset G\left(\mathbb{A}_{f}\right)$ a compact open subgroup, we have an induced action of $\bar{\pi}_{0} \pi(G)$ on the set of irreducible components of $\operatorname{Sh}_{K}(G, X)_{\mathbb{C}}$. All these components have the same stabilizer in $\bar{\pi}_{0} \pi(G)$, namely $q(K)$. Let $\operatorname{rec}_{K}: \operatorname{Gal}\left(E^{\mathrm{ab}} / E\right) \rightarrow \bar{\pi}_{0} \pi(G) / q(K)$ denote the reciprocity map modulo $q(K)$.

3.5 Proposition. Retain the notation and assumptions of 3.1. Then the image of the homomorphism $\phi: \pi_{1}\left(S_{0}\right) \rightarrow K_{0}$ is the subgroup $q^{-1}(\operatorname{Im}(\mathrm{rec})) \cap K_{0}$ of $K_{0}$.

Proof. Since $F$ is defined to be the field of definition of the irreducible component $S_{0, \mathbb{C}} \subset \operatorname{Sh}_{K_{0}}(G, X)_{\mathbb{C}}$, we have

$$
\operatorname{Gal}\left(E^{\mathrm{ab}} / F\right)=\operatorname{rec}^{-1}\left(q\left(K_{0}\right)\right)
$$

as subgroups of $\operatorname{Gal}\left(E^{\mathrm{ab}} / E\right)$. For $K$ a normal open subgroup of $K_{0}$, the set of geometric irreducible components of $S_{K}$ is a torsor under $q\left(K_{0}\right) / q(K) \subset \bar{\pi}_{0} \pi(G) / q(K)$, and the irreducible components of $S_{K}$ (over $F$ ) correspond to the orbits under the action of $\mathrm{Gal}\left(E^{\mathrm{ab}} / F\right)$ via $\operatorname{rec}_{K}$. Hence the image of $\phi_{K}: \pi_{1}\left(S_{0}\right) \rightarrow K_{0} / K$ is the inverse image under $q: K_{0} / K \rightarrow q\left(K_{0}\right) / q(K) \operatorname{of} \operatorname{rec}_{K}\left(\operatorname{Gal}\left(E^{\mathrm{ab}} / F\right)\right)$. The latter group is the image of $\left(\operatorname{Im}(\mathrm{rec}) \cap q\left(K_{0}\right)\right)$ in $q\left(K_{0}\right) / q(K)$, and so we find that $\operatorname{Im}\left(\phi_{K}\right)$ is the image of $q^{-1}(\operatorname{Im}(\mathrm{rec})) \cap K_{0}$ in $K_{0} / K$. The proposition follows by passing to the limit.

3.6 Theorem. With assumptions as in 3.1, the cokernel of the reciprocity map (3.4.1) has finite exponent, and it is a finite discrete group (i.e., $\operatorname{Im}(\mathrm{rec}) \subset \bar{\pi}_{0} \pi(G)$ is an open subgroup) if $(G, X)$ is maximal.

Before we start discussing the proof, let us give the main corollary of this result.

3.7 Corollary. With assumptions as in 3.1, consider the homomorphism $\phi: \pi_{1}\left(S_{0}\right) \rightarrow K_{0}$, and for a prime number $\ell$, let $\phi_{\ell}: \pi_{1}\left(S_{0}\right) \rightarrow K_{0, \ell}$ be its $\ell$-primary component. Fix a free $\mathbb{Z}$-module $H$ of finite rank and a closed embedding $i: G \hookrightarrow \mathrm{GL}(H \otimes \mathbb{Q})$, and let $\mathscr{G} \subset \mathrm{GL}(H)$ be the Zariski closure of $G$ in $\mathrm{GL}(H)$.

(i) There exists a positive integer $N$, depending only on $\mathscr{G}$ and $X$, such that $\left.\left[\mathscr{G}_{(} \mathbb{Z}_{\ell}\right): \operatorname{Im}\left(\phi_{\ell}\right)\right] \leq N$ for all $\ell$. 
(ii) For almost all $\ell$ the image of $\phi_{\ell}$ contains the commutator subgroup of $\mathscr{G}\left(\mathbb{Z}_{\ell}\right)$.

(iii) If $(G, X)$ is maximal in the sense defined in $2.7, \operatorname{Im}(\phi)$ is an open subgroup of $G\left(\mathbb{A}_{f}\right)$.

Proof. In view of Proposition 3.5. (iii) of the corollary is immediate from the second assertion in the theorem. For (ii) we only have to note that $\operatorname{Im}(\phi)$ contains the commutator subgroup of $K_{0}$ and that $K_{0, \ell}=\mathscr{G}\left(\mathbb{Z}_{\ell}\right)$ for almost all $\ell$.

It remains to deduce (i) from the theorem. With the notation of $3.4, q^{-1}(\operatorname{Im}(\mathrm{rec}))$ is a normal subgroup of $G\left(\mathbb{A}_{f}\right)$, with profinite abelian quotient $G\left(\mathbb{A}_{f}\right) / q^{-1}(\operatorname{Im}(\mathrm{rec})) \cong \operatorname{Coker}(\mathrm{rec})$. If $m$ is the exponent of Coker(rec), it follows that $K_{0, \ell} / \operatorname{Im}\left(\phi_{\ell}\right)$ is a compact abelian $\ell$-adic analytic group that is killed by $m$. Hence it is finite. It follows that $\operatorname{Im}\left(\phi_{\ell}\right)$ has finite index in $\mathscr{G}\left(\mathbb{Z}_{\ell}\right)$ for all $\ell$, and so it suffices to prove (i) for all $\ell$ sufficiently large.

Choose a multiple $M$ of $m$ such that $\mathscr{G}$ is reductive over $\mathbb{Z}[1 / M]$ and $K_{0, \ell}=\mathscr{G}\left(\mathbb{Z}_{\ell}\right)$ for all $\ell>M$. Fix an $\ell>M$, and let $\mathscr{G}_{0}=\mathscr{G} \otimes \mathbb{F}_{\ell}$ denote the characteristic $\ell$ fibre of $\mathscr{G}$. Let $\tilde{\mathscr{G}}_{0} \rightarrow \mathscr{G}_{0}^{\text {der }}$ be the simply connected cover of the derived subgroup. The image of $\tilde{\mathscr{G}}_{0}\left(\mathbb{F}_{\ell}\right) \rightarrow \mathscr{G}_{0}^{\text {der }}\left(\mathbb{F}_{\ell}\right)$ is the normal subgroup $\mathscr{G}_{0}\left(\mathbb{F}_{\ell}\right)^{+} \triangleleft \mathscr{G}_{0}\left(\mathbb{F}_{\ell}\right)$ that is generated by the $\ell$-Sylow subgroups.

Still with $\ell>M$, the image of $\phi_{\ell}$ contains the subgroup of $\mathscr{G}\left(\mathbb{Z}_{\ell}\right)$ generated by the $\ell$-Sylow groups, and hence contains all elements $g \in \mathscr{G}\left(\mathbb{Z}_{\ell}\right)$ whose reduction modulo $\ell$ lies in $\mathscr{G}_{0}\left(\mathbb{F}_{\ell}\right)^{+}$. It therefore suffices to bound the $m$-torsion in $\mathscr{G}_{0}\left(\mathbb{F}_{\ell}\right) / \mathscr{G}_{0}\left(\mathbb{F}_{\ell}\right)^{+}$by a constant independent of $\ell$. As a first step, let $\mu$ be the kernel of $\tilde{\mathscr{G}}_{0} \rightarrow \mathscr{G}_{0}^{\text {der }}$; this is a group of multiplicative type whose rank $|\mu|$ only depends on $G$. (If $R$ is the absolute rank of $G$, it is known that $\mu$ has rank at most $2^{R}$.) The quotient $\mathscr{G}_{0}^{\operatorname{der}}\left(\mathbb{F}_{\ell}\right) / \mathscr{G}_{0}\left(\mathbb{F}_{\ell}\right)^{+}$injects into $H^{1}\left(\mathbb{F}_{\ell}, \mu\right)$, which is a quotient of $\mu\left(\overline{\mathbb{F}}_{\ell}\right)$ (cohomology of procyclic groups). In particular, $\left[\mathscr{G}_{0}^{\operatorname{der}}\left(\mathbb{F}_{\ell}\right): \mathscr{G}_{0}\left(\mathbb{F}_{\ell}\right)^{+}\right]$divides $|\mu|$. It therefore suffices to bound the $m$-torsion in $\mathscr{G}_{0}\left(\mathbb{F}_{\ell}\right) / \mathscr{G}_{0}^{\operatorname{der}}\left(\mathbb{F}_{\ell}\right)$ by a constant independent of $\ell$. Writing $\mathscr{G}_{0}^{\text {ab }}=\mathscr{G}_{0} / \mathscr{G}_{0}^{\text {der }}$, the group $\mathscr{G}_{0}\left(\mathbb{F}_{\ell}\right) / \mathscr{G}_{0} \operatorname{der}\left(\mathbb{F}_{\ell}\right)$ is a subgroup of $\mathscr{G}_{0}^{\mathrm{ab}}\left(\mathbb{F}_{\ell}\right)$. Further, if $r$ is the rank of the torus $G^{\mathrm{ab}}$, the kernel of multiplication by $m$ on $\mathscr{G}_{0}^{\text {ab }}$ is a finite group scheme of rank $r^{m}$; hence the $m$-torsion in $\mathscr{G}_{0}^{\text {ab }}\left(\mathbb{F}_{\ell}\right)$ has cardinality at most $r^{m}$.

We now turn to the proof of Theorem 3.6. In 3.9 and 3.11 we first prove the result in two special cases; the general case is then deduced in 3.13. To prove that Coker(rec) has finite exponent (resp., is finite), we use Deligne's group-theoretic description of the reciprocity map. (See [8], Théorème 2.6.3.) If $E=E(G, X)$ is the reflex field, we simply write $E^{*}$ for the $\mathbb{Q}$-torus $\operatorname{Res}_{E / \mathbb{Q}} \mathbb{G}_{\mathrm{m}, E}$. Class field theory gives an isomorphism $\pi_{0} \pi\left(E^{*}\right) \stackrel{\sim}{\rightarrow} \operatorname{Gal}\left(E^{\mathrm{ab}} / E\right)$, which we normalize as in [8], 0.8.

We start with a general remark that is useful to us.

3.8 Remark. Let $f: G_{1} \rightarrow G_{2}$ be a surjective homomorphism of reductive $\mathbb{Q}$-groups. Factor $f$ as

$$
G_{1} \rightarrow G_{2}^{\prime} \stackrel{\psi}{\rightarrow} G_{2},
$$

where $G_{2}^{\prime}=G_{1} / \operatorname{Ker}(f)^{0}$. We make the simplifying assumption that $\operatorname{Ker}(\psi)$ (which is the finite étale group scheme $\left.\pi_{0}(\operatorname{Ker}(f))\right)$ is commutative, as this is the only case we need.

By [15], Proposition $6.5, G_{1}(\mathbb{A}) \rightarrow G_{2}^{\prime}(\mathbb{A})$ has open image. The commutativity of $\operatorname{Ker}(\psi)$ implies that the image of $\psi(\mathbb{A}): G_{2}^{\prime}(\mathbb{A}) \rightarrow G_{2}(\mathbb{A})$ is a normal subgroup. Further, if $M$ is the rank of $\operatorname{Ker}(\psi)$ then $H^{1}\left(\operatorname{Spec}(\mathbb{A})_{\text {ét }}, \operatorname{Ker}(\psi)\right)$, and hence also the cokernel of $\psi(\mathbb{A})$, is killed by $M$.

3.9 The case of a torus. Suppose $G=T$ is a torus, in which case $X=\{h\}$ is a singleton. By definition of the reflex field $E$, the corresponding cocharacter $\mu: \mathbb{G}_{\mathrm{m}, \mathbb{C}} \rightarrow T_{\mathbb{C}}$ is defined over $E$; so we 
have a homomorphism $\mu: \mathbb{G}_{\mathrm{m}, E} \rightarrow T_{E}$. Restricting scalars to $\mathbb{Q}$ and composing with the norm map then gives a homomorphism of algebraic tori

$$
\nu: E^{*} \stackrel{\operatorname{Res}(\mu)}{\longrightarrow} \operatorname{Res}_{E / \mathbb{Q}} T_{E} \stackrel{\operatorname{Norm}_{E / \mathbb{Q}}}{\longrightarrow} T .
$$

Via the class field isomorphism, the reciprocity map (3.4.1) is the inverse of the composition

$$
\pi_{0} \pi\left(E^{*}\right) \stackrel{\pi_{0} \pi(\nu)}{\longrightarrow} \pi_{0} \pi(T) \stackrel{\mathrm{pr}}{\longrightarrow} \bar{\pi}_{0} \pi(T) .
$$

We apply what was explained in 2.2 taking $M=T$. In this case $\pi_{1}(T)$ is just the cocharacter group $X_{*}(T)$. With $\Gamma=\operatorname{Gal}(\overline{\mathbb{Q}} / \mathbb{Q})$, the assumption that $T$ is the Mumford-Tate group of $h$ means that $\mathbb{Z}[\Gamma] \cdot \mu$ has finite index in $X_{*}(T)$. Further, $(T,\{h\})$ is maximal if and only if $X_{*}(T)$ is generated by $\mu$ as a $\mathbb{Z}[\Gamma]$-module.

On the other hand, by definition of the reflex field $E$, the stabilizer of $\mu \in X_{*}(T)$ in $\Gamma$ is precisely the subgroup $\Gamma_{E}=\operatorname{Gal}(\overline{\mathbb{Q}} / E) \subset \Gamma$. If $\mathfrak{a} \subset \mathbb{Z}[\Gamma]$ is the left ideal generated by the augmentation ideal of $\mathbb{Z}\left[\Gamma_{E}\right]$, we have $X_{*}\left(E^{*}\right) \cong \mathbb{Z}[\Gamma] / \mathfrak{a}$ as Galois modules, and $X_{*}(\nu): X_{*}\left(E^{*}\right) \rightarrow X_{*}(T)$ is the map given by $(\gamma \bmod \mathfrak{a}) \mapsto \gamma \cdot \mu$. From the preceding remarks it therefore follows that $\nu$, as a homomorphism of algebraic tori, is surjective, and that $\operatorname{Ker}(\nu)$ is connected if $(T,\{h\})$ is Hodge-maximal. The assertion of 3.6 now follows from Remark 3.8.

3.10 Let $f:\left(G_{1}, X_{1}\right) \rightarrow\left(G_{2}, X_{2}\right)$ be a morphism of Shimura data. Let $E_{i}(i=1,2)$ be the reflex field of $\left(G_{i}, X_{i}\right)$, and denote by $E_{i}^{\mathrm{ab}}$ its maximal abelian extension. Then $E_{1}$ is a finite extension of $E_{2}$ in $\mathbb{C}$ and we have a commutative diagram

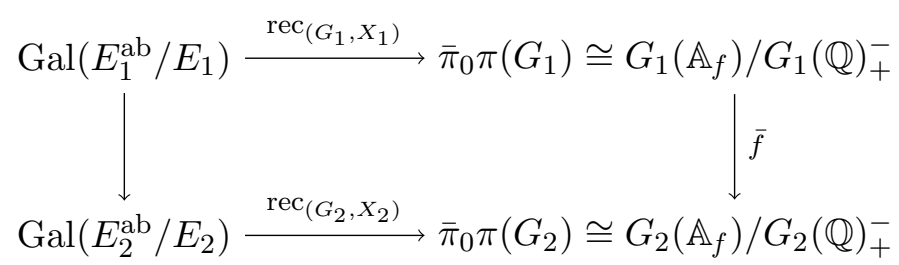

in which $\bar{f}$ denotes the map induced by $f$. The image of the left vertical map is a subgroup of finite index in $\operatorname{Gal}\left(E_{2}^{\mathrm{ab}} / E_{2}\right)$.

3.11 The case when $G^{\text {der }}$ is simply connected. Next we treat the case when the derived group $G^{\text {der }}$ is simply connected. Let $G^{\text {ab }}=G / G^{\text {der }}$ and let $p: G \rightarrow G^{\text {ab }}$ be the canonical map. Then $h^{\mathrm{ab}}=p \circ h$ is independent of $h \in X$, and $\left(G^{\mathrm{ab}},\left\{h^{\mathrm{ab}}\right\}\right)$ is a Shimura datum. By Remark 2.8(ii), if $(G, X)$ is maximal, so is $\left(G^{\mathrm{ab}}, X^{\mathrm{ab}}\right)$. We apply 3.10 to the morphism $p:(G, X) \rightarrow\left(G^{\mathrm{ab}},\left\{h^{\mathrm{ab}}\right\}\right)$. By [7, Théorème 2.4, the right vertical map in the diagram is surjective with finite kernel. (Deligne's result says that $p$ induces an isomorphism on the groups that we denote by $\pi_{0} \pi$; the groups $\bar{\pi}_{0} \pi$ are quotients of these by finite subgroups.) The theorem for $(G, X)$ therefore follows from the result for $\left(G^{\mathrm{ab}}, X^{\mathrm{ab}}\right)$, which was proven in 3.9 .

3.12 Lemma. Let $(G, X)$ be a Shimura datum as in 3.1. Then there exists a Shimura datum $(\tilde{G}, \tilde{X})$ and a morphism $f:(\tilde{G}, \tilde{X}) \rightarrow(G, X)$ such that

(a) the group $\tilde{G}$ is the generic Mumford-Tate group on $\tilde{X}$;

(b) the homomorphism $f: \tilde{G} \rightarrow G$ is surjective, and the induced $f^{\mathrm{der}}: \tilde{G}^{\mathrm{der}} \rightarrow G^{\mathrm{der}}$ is the simply connected cover of $G^{\text {der }}$. 
Moreover, if $(G, X)$ is maximal, we can choose $(\tilde{G}, \tilde{X})$ such that it is maximal, too, and such that the kernel of $f: \tilde{G} \rightarrow G$ is connected.

Proof. By [13], Application 3.4, there exists a morphism of Shimura data $f_{1}:\left(G_{1}, \tilde{X}\right) \rightarrow(G, X)$ such that $f_{1}: G_{1} \rightarrow G$ is surjective, $\operatorname{Ker}\left(f_{1}\right)$ is a torus, and $f_{1}^{\text {der }}: G_{1}^{\text {der }} \rightarrow G^{\text {der }}$ is the simply connected cover. Let $\tilde{G} \subset G_{1}$ be the generic Mumford-Tate group on $\tilde{X}$, and let $f: \tilde{G} \rightarrow G$ be the restriction of $f_{1}$ to $\tilde{G}$. The assumption that $G$ is the generic Mumford-Tate group on $X$ implies that $f$ is surjective, and as $\tilde{G}$ is normal in $G_{1}$, this implies that $\tilde{G}^{\text {der }}=G_{1}^{\text {der }}$. So $(\tilde{G}, \tilde{X})$ and $f$ satisfy (a) and (b).

Next assume $(G, X)$ is maximal. We claim that for any $f:(\tilde{G}, \tilde{X}) \rightarrow(G, X)$ such that (a) and (b) hold, $\operatorname{Ker}(f)$ is connected (hence a torus). Indeed, let $\left(G_{2}, X_{2}\right)$ be the quotient of $(\tilde{G}, \tilde{X})$ by $\operatorname{Ker}(f)^{0}$. We then have an induced morphism of Shimura data $\left(G_{2}, X_{2}\right) \rightarrow(G, X)$. The map $G_{2} \rightarrow G$ is an isogeny with kernel the group scheme $\pi_{0}(\operatorname{Ker}(f))$ of connected components of $\operatorname{Ker}(f)$. By maximality of $(G, X)$ this implies that $\pi_{0}(\operatorname{Ker}(f))$ is trivial, which proves the claim.

The Shimura datum $(\tilde{G}, \tilde{X})$ we have obtained need not be maximal. By Remark 2.8(iii), there exists an isogeny $(\hat{G}, \hat{X}) \rightarrow(\tilde{G}, \tilde{X})$ with $(\hat{G}, \hat{X})$ maximal, and by 2.8(i) $\hat{G}$ is the generic Mumford-Tate

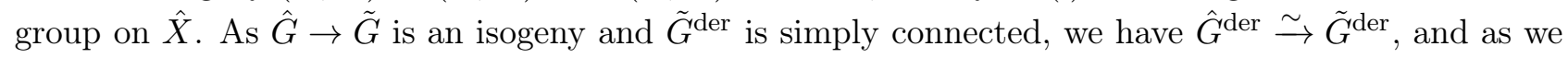
have already seen, the maximality of $(G, X)$ implies that the kernel of $\hat{G} \rightarrow G$ is connected.

3.13 To complete the proof of Theorem 3.6, let $(G, X)$ be the Shimura datum considered in the assertion, and take $f:(\tilde{G}, \tilde{X}) \rightarrow(G, X)$ as in Lemma 3.12, As shown in 3.11, the theorem is true for $(\tilde{G}, \tilde{X})$. In view of diagram (3.10.1), it suffices to show that the induced map $\tilde{G}\left(\mathbb{A}_{f}\right) \rightarrow G\left(\mathbb{A}_{f}\right)$ has cokernel of finite exponent, and that the image of this map is open if $(G, X)$ is maximal. This follows from Remark 3.8 .

\section{Galois-generic points}

4.1 Let $S$ be a geometrically connected scheme of finite type over a field $k$. Assume given a continuous representation $\psi: \pi_{1}(S) \rightarrow G\left(\mathbb{A}_{f}\right)$, where $G$ is an algebraic group over $\mathbb{Q}$. (Throughout, we omit the choice of a geometric base point of $S$ from the notation.) If $\ell$ is a prime number, we denote by $\psi_{\ell}: \pi_{1}(S) \rightarrow G\left(\mathbb{Q}_{\ell}\right)$ the $\ell$-primary component of $\psi$.

If $y$ is a point of $S$, we have a homomorphism $\sigma_{y}: \pi_{1}(y) \rightarrow \pi_{1}(S)$, well-determined up to conjugation. (Recall that $\pi_{1}(y)$ is the absolute Galois group of the residue field $k(y)$.)

4.2 Definition. (i) A point $y \in S$ is said to be Galois-generic with respect to $\psi$ if the image of $\psi \circ \sigma_{y}$ is open in the image of $\psi$.

(ii) A point $y \in S$ is said to be $\ell$-Galois-generic with respect to $\psi$ if the image of $\psi_{\ell} \circ \sigma_{y}$ is open in the image of $\psi_{\ell}$.

If it is clear which $\psi$ we mean, we omit the phrase "with respect to $\psi$ ".

Clearly, if a point $y$ is Galois-generic, it is $\ell$-Galois generic for all $\ell$. The following theorem by the first author and Kret (see [6], Theorem 1.1) shows that for the representation associated with a Shimura variety of abelian type, the converse holds.

4.3 Theorem. Let $(G, X)$ be a Shimura datum of abelian type, $K_{0} \subset G\left(\mathbb{A}_{f}\right)$ a neat compact open subgroup, $h_{0} \in X$ a base point. Let $\phi_{\bar{s}}: \pi_{1}\left(S_{0}\right) \rightarrow K_{0}$ be the associated representation, as defined in 3.1 . If a point $y \in S$ is $\ell$-Galois-generic for some prime number $\ell$ then $y$ is Galois-generic. 


\section{Application to abelian varieties}

5.1 For $g \geq 1$, equip $\mathbb{Z}^{2 g}$ with the standard symplectic form, and let $\mathrm{GSp}_{2 g}$ be the reductive group over $\mathbb{Z}$ of symplectic similitudes of $\mathbb{Z}^{2 g}$. Let $\mathfrak{H}_{g}^{ \pm}$be the set of homomorphisms $h: \mathbb{S} \rightarrow \mathrm{GSp}_{2 g, \mathbb{R}}$ that define a Hodge structure of type $(-1,0)+(0,-1)$ on $\mathbb{Z}^{2 g}$ for which $\pm 2 \pi i \cdot \psi$ is a polarization. The real group $\mathrm{GSp}_{2 g}(\mathbb{R})$ acts transitively on $\mathfrak{H}_{g}^{ \pm}$, and the pair $\left(\mathrm{GSp}_{2 g}, \mathfrak{H}_{g}^{ \pm}\right)$is a Shimura datum with reflex field $\mathbb{Q}$.

Let $K(3) \subset \mathrm{GSp}_{2 g}(\hat{\mathbb{Z}})$ be the subgroup of elements that reduce to the identity modulo 3 . Then $\mathrm{Sh}_{K(3)}\left(\mathrm{GSp}_{2 g}, \mathfrak{H}_{g}^{ \pm}\right)$is isomorphic (over $\mathbb{Q}$ ) to the moduli space $\mathscr{A}_{g, 3}$ of $g$-dimensional principally polarized abelian varieties with a (Jacobi) level 3 structure. See for instance [7, Section 4. In what follows we identify the two schemes.

Let $(B, \lambda)$ be a principally polarized abelian variety of dimension $g$ over a subfield $k \subset \mathbb{C}$ that is finitely generated over $\mathbb{Q}$. Assume that all 3 -torsion points of $B$ are $k$-rational. (This implies that $\mathbb{Q}\left(\zeta_{3}\right) \subset k$.) Choose a similitude $i: H_{1}(B(\mathbb{C}), \mathbb{Z}) \stackrel{\sim}{\rightarrow} \mathbb{Z}^{2 g}$. This gives a Hodge structure on $\mathbb{Z}^{2 g}$; let $h_{0} \in \mathfrak{H}_{g}^{ \pm}$be the corresponding point. As in section 3.1, $\left[h_{0}, e K(3)\right]$ defines a $\mathbb{C}$-valued point $\bar{t}_{0} \in \mathscr{A}_{g, 3}(\mathbb{C})$. The corresponding level 3 structure on $\left(B_{\mathbb{C}}, \lambda\right)$ is defined over $k$, which means that $\bar{t}_{0}$ comes from a $k$-valued point $t_{0} \in \mathscr{A}_{g, 3}(k)$ by composing it with the given embedding $k \hookrightarrow \mathbb{C}$.

Let $\mathscr{A}_{0} \subset \mathscr{A}_{g, 3} \otimes \mathbb{Q}\left(\zeta_{3}\right)$ be the irreducible component such that $t_{0} \in \mathscr{A}_{0}(k)$. This component is geometrically irreducible. The construction of 3.1 gives a representation

$$
\phi_{\bar{t}}: \pi_{1}\left(\mathscr{A}_{0}, \bar{t}_{0}\right) \rightarrow K(3) \subset \mathrm{GL}_{2 g}(\hat{\mathbb{Z}}) .
$$

(Here, as in 3.1, $\bar{t}$ refers to the compatible system of base points $\left(\bar{t}_{K}\right)$ obtained from $h_{0}$.) The point $t_{0}$ gives rise to a homomorphism $\sigma_{t_{0}}: \operatorname{Gal}(\bar{k} / k) \rightarrow \pi_{1}\left(\mathscr{A}_{0}, \bar{t}_{0}\right)$ such that the composition with the projection $\pi_{1}\left(\mathscr{A}_{0}, \bar{t}_{0}\right) \rightarrow \operatorname{Gal}\left(\overline{\mathbb{Q}} / \mathbb{Q}\left(\zeta_{3}\right)\right)$ is the natural homomorphism $\operatorname{Gal}(\bar{k} / k) \rightarrow \operatorname{Gal}\left(\overline{\mathbb{Q}} / \mathbb{Q}\left(\zeta_{3}\right)\right)$. Note that $\sigma_{t_{0}}$ is canonically defined, not only up to conjugation.

On the other hand, if we let $H=H_{1}(B(\mathbb{C}), \mathbb{Z})$ then we may identify the full Tate module $\hat{H}=\lim _{n} B[n](\bar{k})$ (limit over all positive integers $n$, partially ordered by divisibility) with $H \otimes \hat{\mathbb{Z}}$. Via the chosen similitude $i$ we obtain an isomorphism $\hat{H} \stackrel{\sim}{\rightarrow} \hat{\mathbb{Z}}^{2 g}$. The natural Galois action on $\hat{H}$ therefore gives a representation

$$
\rho_{B}: \operatorname{Gal}(\bar{k} / k) \rightarrow \mathrm{GL}_{2 g}(\hat{\mathbb{Z}}) .
$$

The following result is an immediate consequence of the definitions and the modular interpretation of $\mathrm{Sh}\left(\mathrm{GSp}_{2 g}, \mathfrak{H}_{g}^{ \pm}\right)$. See also [24], Remark 2.8, as well as the next section, where we discuss the analogous (but slightly more involved) case of K3 surfaces.

5.2 Proposition. The representations $\phi_{\bar{t}} \circ \sigma_{t_{0}}$ and $\rho_{B}$ are the same.

The main result of this section is that the usual form of the Mumford-Tate conjecture implies the integral and adelic versions of the Mumford-Tate conjecture as formulated in 1.3 and 2.5 .

5.3 Theorem. Let $B$ be an abelian variety over a subfield $k \subset \mathbb{C}$ that is finitely generated over $\mathbb{Q}$. Assume that for some prime number $\ell$ the Mumford-Tate conjecture for $B$ is true. Then the integral and adelic Mumford-Tate conjectures for $B$ are true as well.

Note that in this case the last part of Conjecture 1.3 says that the image of $\rho_{B}$ contains an open subgroup of $\hat{\mathbb{Z}}^{*} \cdot$ id. This is in fact a result proven by Wintenberger [28]. (The result is stated in loc. cit. only for $k$ a number field; this implies the same result over finitely generated fields, as we can 
specialize $B$ to an abelian variety over a number field in such a way that the Mumford-Tate group does not change.)

Proof. In proving the theorem, we may replace the ground field $k$ with a finite extension and $B$ with an isogenous abelian variety. Hence we may assume that $B$ admits a principal polarization and that all 3-torsion points of $B$ are $k$-rational. This puts us in the situation of 5.1. We retain the notation and choices introduced there.

Via the chosen similitude $H \stackrel{\sim}{\rightarrow} \mathbb{Z}^{2 g}$ we may view the Mumford-Tate group $G_{\mathrm{B}}$ of $B$ as an algebraic subgroup of $\mathrm{GSp}_{2 g, \mathbb{Q}}$. We take its Zariski closure $G_{\mathrm{B}} \subset \mathrm{GSp}_{2 g}$ as integral model. (There is no need to introduce new notation for this integral form.) With $h_{0} \in \mathfrak{H}_{g}^{ \pm}$as in 5.1, let $X \subset \mathfrak{H}_{g}^{ \pm}$be the $G_{\mathrm{B}}(\mathbb{R})$-orbit of $h_{0}$. The pair $\left(G_{\mathrm{B}}, X\right)$ is a Shimura datum, and by construction we have a morphism $f:\left(G_{\mathrm{B}}, X\right) \rightarrow\left(\mathrm{GSp}_{2 g}, \mathfrak{H}_{g}^{ \pm}\right)$. Let $K_{0}=f^{-1}(K(3))$, which is a neat compact open subgroup of $G_{\mathrm{B}}\left(\mathbb{A}_{f}\right)$, and, with $E$ the reflex field of $\left(G_{\mathrm{B}}, X\right)$, let $\mathrm{Sh}(f): \mathrm{Sh}_{K_{0}}\left(G_{\mathrm{B}}, X\right) \rightarrow \mathscr{A}_{g, 3} \otimes E$ be the morphism induced by $f$.

Let $\bar{s}_{0}=\left[h_{0}, e K_{0}\right]$, which is a $\mathbb{C}$-valued point of $\operatorname{Sh}_{K_{0}}\left(G_{\mathrm{B}}, X\right)$ whose image under $\operatorname{Sh}(f)$ is $\bar{t}_{0}$. As in 3.1, let $S_{0, \mathbb{C}} \subset \mathrm{Sh}_{K_{0}}\left(G_{\mathrm{B}}, X\right)_{\mathbb{C}}$ be the irreducible component containing $\bar{s}_{0}$, let $F \subset \mathbb{C}$ be its field of definition, and let $S_{0} \subset \mathrm{Sh}_{K_{0}}$ be the geometrically irreducible component thus obtained. Possibly after replacing $k$ with a finite extension, $\bar{s}_{0}$ comes from a point $s_{0} \in S_{0}(k)$, whose image in $\mathscr{A}_{g, 3}(k)$ is the point $t_{0}$ that corresponds to $(B, \lambda)$ equipped with a suitable level 3 structure. This point $s_{0}$ gives rise to a homomorphism $\sigma_{s_{0}}: \operatorname{Gal}(\bar{k} / k) \rightarrow \pi_{1}\left(S_{0}, \bar{s}_{0}\right)$. With $\phi_{\bar{s}}: \pi_{1}\left(S_{0}, \bar{s}_{0}\right) \rightarrow K_{0} \subset G_{\mathrm{B}}(\hat{\mathbb{Z}})$ the homomorphism (3.1.1), it follows from the functoriality explained in Remark 3.2 together with Proposition 5.2 that $\phi_{\bar{s}} \circ \sigma_{s_{0}}$ is the Galois representation on the (full) Tate module of $B$.

Let $\ell$ be a prime number and $\phi_{\ell}: \pi_{1}\left(S_{0}, \bar{s}_{0}\right) \rightarrow G_{\mathrm{B}}\left(\mathbb{Z}_{\ell}\right)$ the $\ell$-primary component of $\phi$. If the Mumford-Tate conjecture for $B$ is true at $\ell, s_{0}$ is $\ell$-Galois-generic with respect to the representation $\phi$. (See the remark after 1.3.) By Theorem 4.3, $s_{0}$ is then Galois-generic, and in view of the description of $\operatorname{Im}(\phi)$ given in Corollary 3.7, this implies that Conjecture 1.3 and, if the Hodge structure $H$ is maximal, Conjecture 2.5 are true for $B$.

5.4 Remarks. (i) There are many special classes of abelian varieties for which the Mumford-Tate conjecture is known. For a sample of such results, see for instance [14, Section 5, or the more recent [12] and the references contained therein. On the other hand, already for abelian varieties of dimension 4 the Mumford-Tate conjecture remains open.

(ii) When we restrict our attention to the image of $\rho$ intersected with the derived subgroup of $G_{\mathrm{B}}$, a result related to our Theorem 5.3 was obtained by Hui and Larsen; see [9], Theorem 4.2. Their work is based on a very different approach.

5.5 Example. As a first application we recover the result given in [21], 11.11. If $B$ is a $g$-dimensional abelian variety with $g$ odd (or $g=2$, or $g=4$ ) and $\operatorname{End}\left(B_{\bar{k}}\right)=\mathbb{Z}$, it is known that the Mumford-Tate conjecture for $B$ is true and that the Mumford-Tate group is the full $\mathrm{GSp}_{2 g}$. (See [14], Theorem 5.14, for a more general result.) It is easily seen that the Shimura datum $\left(\mathrm{GSp}_{2 g}, \mathfrak{H}_{g}^{ \pm}\right)$is maximal; the conclusion therefore is that in this case the image of the representation $\rho_{B}$ is open in $\operatorname{GSp}_{2 g}\left(\mathbb{A}_{f}\right)$.

Next we want to give examples of abelian varieties $B$ over finitely generated subfields of $\mathbb{C}$ for which the Hodge structure $H_{1}(B(\mathbb{C}), \mathbb{Q})$ is not Hodge-maximal. Such examples of course also give us Shimura data of Hodge type that are not maximal. The first examples we discuss are of CM type; after that, we discuss an example in which the Mumford-Tate group is the almost direct product of 
the homotheties and a semisimple group.

5.6 Example. For our first construction, we start with a totally real field $E_{0}$ of degree $g$ over $\mathbb{Q}$. Let $\sigma_{1}, \ldots, \sigma_{g}$ be the complex embeddings of $E_{0}$. Let $k$ be an imaginary quadratic field. Then $E=k \cdot E_{0}$ is a CM field. Fix an embedding $\alpha: k \rightarrow \mathbb{C}$, and let $\tau_{i}(i=1, \ldots, g)$ be the complex embedding of $E$ that extends $\sigma_{i}$ and such that $\left.\tau_{i}\right|_{k}=\alpha$. Thus, $T=\left\{\tau_{1}, \ldots, \tau_{g}, \bar{\tau}_{1}, \ldots, \bar{\tau}_{g}\right\}$ is the set of complex embeddings of $E$.

Consider the CM type $\Phi$ on $E$ given by

$$
\Phi=\left\{\tau_{1}, \bar{\tau}_{2}, \ldots, \bar{\tau}_{g}\right\}
$$

The pair $(E, \Phi)$ gives rise to an isogeny class of $g$-dimensional complex abelian varieties $B$, determined by the rule that $H_{1}(B(\mathbb{C}), \mathbb{Q}) \cong E$ as a $\mathbb{Q}$-vector space, with Hodge decomposition of $H_{1}(B(\mathbb{C}), \mathbb{C}) \cong$ $\oplus_{\tau \in T} \mathbb{C}^{(\tau)}$ given by the rule that $\mathbb{C}^{(\tau)}$ is of type $(-1,0)$ if $\tau \in \Phi$ and of type $(0,-1)$ otherwise. If $g>1$ then $\Phi$ is a primitive CM type; in this case $B$ is simple. As any abelian variety of CM type, $B$ is defined over a number field, and by a result of Pohlmann [16] the Mumford-Tate conjecture is true for $B$.

As in Section 3, if $F$ is a number field we simply write $F^{*}$ for the torus $\operatorname{Res}_{F / \mathbb{Q}} \mathbb{G}_{\mathrm{m}, F}$. Let Norm: $E^{*} \rightarrow E_{0}^{*}$ be the norm homomorphism, and let $U \subset E^{*}$ be the subtorus given by $U=$ $\operatorname{Norm}^{-1}\left(\mathbb{Q}^{*}\right)$. The cocharacter group $X_{*}\left(E^{*}\right)$ is the free $\mathbb{Z}$-module on the set $T$. The cocharacter group of $U$ is given by

$$
X_{*}(U)=\left\{\sum_{i=1}^{g} a_{i} \tau_{i}+\sum_{i=1}^{g} b_{i} \bar{\tau}_{i} \in X_{*}\left(E^{*}\right) \mid a_{i}+b_{i} \text { is independent of } i\right\} .
$$

The elements $f_{i}=\tau_{i}-\bar{\tau}_{i}(i=1, \ldots, g)$ together with $f_{g+1}=\sum_{i=1}^{g} \bar{\tau}_{i}$ form a basis for $X_{*}(U)$.

The cocharacter $\mu: \mathbb{G}_{\mathrm{m}, \mathbb{C}} \rightarrow E^{*}$ corresponding to the Hodge structure $H_{1}(B(\mathbb{C}), \mathbb{Q})$ is given by $\mu=\tau_{1}+\bar{\tau}_{2}+\cdots+\bar{\tau}_{g}=f_{1}+f_{g+1}$. The Galois conjugates of $\mu$ are the elements $f_{i}+f_{g+1}$ for $i=1, \ldots, g$ together with their complex conjugates $f_{1}+\cdots+\hat{f}_{i}+\cdots+f_{g}+f_{g+1}$, for $i=1, \ldots, g$. These are cocharacters in $X_{*}(U)$, and for $g>2$ they span a submodule of index $g-2$ in $X_{*}(U)$. The conclusion, therefore, is that $U$ is the Mumford-Tate group of $B$ if $g>2$, and that $H_{1}(B(\mathbb{C}), \mathbb{Q})$ is not Hodgemaximal if $g>3$. In this last case, the image of the adelic Galois representation is therefore not open in the adelic points of the Mumford-Tate group.

5.7 Example. For our final example, we consider a Shimura datum $(G, X)$ such that

(a) $G$ is an inner form of a split group;

(b) $\pi_{1}(G)$ is non-cyclic.

Note that (b) holds if $G^{\text {ab }}$ has dimension at least 2 , or if $\operatorname{dim}\left(G^{\mathrm{ab}}\right)=1$ and $G^{\text {der }}$ is not simplyconnected. (In the latter case this follows using [3], Cor. 1.7.) If $($ a) holds, $\operatorname{Gal}(\overline{\mathbb{Q}} / \mathbb{Q})$ acts trivially on $\pi_{1}(G)$ (see [3], Lemma 1.8), and by what was explained in 2.2 and 2.7, we conclude that any $(G, X)$ satisfying (a) and (b) is non-maximal.

To obtain a concrete example, let $D$ be a quaternion algebra over $\mathbb{Q}$ that is non-split at infinity, i.e., $D \otimes_{\mathbb{Q}} \mathbb{R}$ is Hamilton's quaternion algebra $\mathbb{H}$. The canonical involution $*$ on $D$ is then a positive involution. Let $n=2 r$ be an even positive integer with $n \geq 6$, and consider a free (left-) $D$-module $V$ of rank $n$ equipped with a (-1)-hermitian form $\Psi$ of discriminant 1 and Witt index $r$. Let $G^{\prime}=\mathrm{U}_{D}(V, \Psi)$ be the corresponding unitary group, which we view as an algebraic subgroup of $\mathrm{GL}_{\mathbb{Q}}(V)$, and let $G \subset \mathrm{GL}_{\mathbb{Q}}(V)$ be the algebraic group generated by $G^{\prime}$ together with the homotheties $\mathbb{G}_{\mathrm{m}} \cdot \mathrm{id}$. The 
group $G^{\prime} \otimes \mathbb{R}$ is isomorphic to the identity component of $\mathrm{U}_{n}^{*}(\mathbb{H})$ (which in some literature is denoted by $\mathrm{SO}^{*}(2 n)$ ), and there is a unique $G(\mathbb{R})$-conjugacy class of homomorphisms $h: \mathbb{S} \rightarrow G_{\mathbb{R}}$ that make the pair $(G, X)$ a Shimura datum of PEL type. (Cf. [8], Section 1.3.) The corresponding Shimura variety parametrizes polarized abelian varieties of dimension $2 n$ with an action by (an order in) $D$, which is of Albert Type III. As $G^{\prime}$ is a $\mathbb{Q}$-simple group, $G$ is the generic Mumford-Tate group on $X$.

Our assumption that $\Psi$ has trivial discriminant implies that the index of $G^{\prime}$ is ${ }^{1} \mathrm{D}_{n}$; see [23], Table II, pages 56-57. This means that $G^{\prime}$ (and hence also $G$ ) is an inner form of the split form, i.e., condition (a) is satisfied. On the other hand, $\operatorname{dim}\left(G^{\mathrm{ab}}\right)=1$ and $G^{\text {der }}$ is not simply-connected, so also (b) is satisfied. (In fact, $\pi_{1}(G) \cong \mathbb{Z} \times(\mathbb{Z} / 2 \mathbb{Z})$.) We conclude that $(G, X)$ is not maximal. If $B$ is a complex abelian variety that corresponds to a Hodge-generic point of the Shimura variety defined by $(G, X)$, the Hodge structure $H_{1}(B(\mathbb{C}), \mathbb{Q})$ is not Hodge-maximal.

\section{Application to K3 surfaces}

6.1 Let $L$ be a number field, $G$ a connected reductive group over $L$, and let $M=\operatorname{Res}_{L / \mathbb{Q}} G$. Then $M_{\mathbb{C}} \cong \prod_{\sigma \in \Sigma} G_{\sigma}$, where $\Sigma$ is the set of complex embeddings of $L$ and $G_{\sigma}=G \otimes_{L, \sigma} \mathbb{C}$. With $\Gamma=$ $\operatorname{Gal}(\overline{\mathbb{Q}} / \mathbb{Q})$ and $\Gamma_{L}=\operatorname{Gal}(\overline{\mathbb{Q}} / L)$, the fundamental group $\pi_{1}(M)$ is the $\Gamma$-module obtained from the $\Gamma_{L}$-module $\pi_{1}(G)$ by induction.

Let $\mu$ be a complex cocharacter of $M$. As in 2.2 , its conjugacy class $\mathscr{C}$ defines an element $[\mathscr{C}] \in \pi_{1}(M)$. Let $W \subset \pi_{1}(M)$ be the $\mathbb{Z}[\Gamma]$-submodule generated by $[\mathscr{C}]$.

Suppose there is a unique $\tau \in \Sigma$ such that the projection $\mu_{\tau}$ of $\mu$ onto the factor $G_{\tau}$ is non-trivial. View $L$ as a subfield of $\mathbb{C}$ via $\tau$; then $\mu_{\tau}$ is a complex cocharacter of $G$. Its conjugacy class $\mathscr{C}_{\tau}$ defines an element $\left[\mathscr{C}_{\tau}\right]$ in $\pi_{1}(G)$. Let $W_{\tau} \subset \pi_{1}(G)$ be the $\mathbb{Z}\left[\Gamma_{L}\right]$-submodule that it generates. In this situation we have $W=\mathbb{Z}[\Gamma] \otimes_{\mathbb{Z}\left[\Gamma_{L}\right]} W_{\tau}$ as submodule of $\pi_{1}(M)=\mathbb{Z}[\Gamma] \otimes_{\mathbb{Z}\left[\Gamma_{L}\right]} \pi_{1}(G)$. Consequently, $\mu$ is maximal as a cocharacter of $M$ if and only if $\mu_{\tau}$ is maximal as a complex cocharacter of $G$.

6.2 Proposition. Let $V$ be a polarizable $\mathbb{Q}$-Hodge structure of $K 3$ type, by which we mean that $V$ is of type $(-1,1)+(0,0)+(1,-1)$ with Hodge numbers $1-n-1$ for some $n$. Then $V$ is Hodge-maximal.

Proof. Without loss of generality we may assume that $V$ is simple as a Hodge structure, which in this case means that there are no non-zero Hodge classes in $V$. (By definition, Hodge-maximality only depends on the Mumford-Tate group $M$ and the defining homomorphism $h: \mathbb{S} \rightarrow M_{\mathbb{R}}$; these do not change if we replace $V$ with $V \oplus \mathbb{Q}(0)$.) Let $L=\operatorname{End}_{\mathbb{Q H S}}(V)$ be the endomorphism algebra of $V$ as a $\mathbb{Q}$-Hodge structure, and choose a polarization form $\psi: V \times V \rightarrow \mathbb{Q}$. As shown by Zarhin in [29], $L$ is a field which is either totally real or a CM field.

First suppose $L$ is totally real. By [25], Lemma 3.2, $\operatorname{dim}_{L}(V) \geq 3$. By [29], Theorem 2.2.1, $M=\operatorname{Res}_{L / \mathbb{Q}} \mathrm{SO}_{L}(V, \Psi)$, where $\Psi: V \times V \rightarrow L$ is the unique symmetric $L$-bilinear form on $V$ such that trace $_{L / \mathbb{Q}} \circ \Psi=\psi$. In particular, $M$ is semisimple. If $\operatorname{dim}_{L}(V)$ is odd, $M$ is simply connected and there is nothing to prove. Next suppose $\operatorname{dim}_{L}(V)=2 l$ is even. Let $\Sigma$ be the set of complex embeddings of $L$. Write $G=\mathrm{SO}_{L}(V, \Psi)$ and let $\mu: \mathbb{G}_{\mathrm{m}, \mathbb{C}} \rightarrow M_{\mathbb{C}} \cong \prod_{\sigma \in \Sigma} G_{\sigma}$ be the cocharacter that gives the Hodge structure. There is a unique $\tau \in \Sigma$ such that $\mu_{\tau} \neq 1$, so we are in the situation of 6.1. The root system of $G_{\mathbb{C}}$ is of type $\mathrm{D}_{l}$, and we follow the notation of [4], Planche IV. Note that the root system in this case is self-dual; further, the calculation that follows goes through without changes if $l=2$. With respect to the basis $\varepsilon_{1}, \ldots, \varepsilon_{l}$ for $\mathbb{R}^{l}=X_{*}(G) \otimes \mathbb{R}$, we have $X_{*}(G)=\mathbb{Z}^{l}$, and the coroot lattice $Q\left(R^{\vee}\right)$ consists of the vectors $\left(m_{1}, \ldots, m_{l}\right) \in \mathbb{Z}^{l}$ for which $\sum m_{j}$ is even. On the other hand, 
the cocharacter $\mu_{\tau}$ corresponds to the vector $(1,0, \ldots, 0)$; its image in $\pi_{1}(G)=X_{*}(G) / Q\left(R^{\vee}\right) \cong \mathbb{Z} / 2 \mathbb{Z}$ is therefore the non-trivial class. By what was explained in 2.2 this implies the assertion.

Next suppose $L$ is a CM field. Let $L_{0} \subset L$ be the totally real subfield. There is a unique symmetric hermitian form $\Psi: V \times V \rightarrow L$ (with respect to complex conjugation on $L$ ) such that trace $L / \mathbb{Q} \circ \Psi=\psi$, and by [29], Theorem 2.3.1, $M=\operatorname{Res}_{L_{0} / \mathbb{Q}} \mathrm{U}_{L}(V, \Psi)$. Write $G=\mathrm{U}_{L}(V, \Psi)$, and let $\Sigma$ be the set of complex embeddings of $L_{0}$. As in the totally real case there is a unique $\tau \in \Sigma$ such that the cocharacter $\mu$ is non-trivial on the factor $G_{\tau}$. If $n=\operatorname{dim}_{L}(V)$, we have $G_{\mathbb{C}} \cong \mathrm{GL}_{n}$ in such a way that $\mu_{\tau}$ is conjugate to the cocharacter $\mathbb{G}_{\mathrm{m}} \rightarrow \mathrm{GL}_{n}$ given by $z \mapsto \operatorname{diag}(z, 1, \ldots, 1)$. It is straightforward to check that the corresponding class in $\pi_{1}\left(\mathrm{GL}_{n}\right) \cong \mathbb{Z}$ is a generator, and again by 2.2 and 6.1 this implies the assertion.

6.3 Remark. In the proposition it is essential that we work with a Hodge structure of weight 0 . As is well-known, if $Y$ is a complex $\mathrm{K} 3$ surface, the Hodge structure $H=H_{\text {prim }}^{2}(Y(\mathbb{C}), \mathbb{Q})$ is not, in general, Hodge-maximal; but $H(1)=H \otimes \mathbb{Q}(1)$ is. For instance, if $\operatorname{End}_{\mathbb{Q} H S}(H)=\mathbb{Q}$, the Mumford-Tate group of $H$ is the group $\operatorname{GO}(H, \phi)$ of orthogonal similitudes, where $\phi$ is a polarization form. We have a non-trivial isogeny $\operatorname{CSpin}(H, \phi) \rightarrow \mathrm{GO}(H, \phi)$, such that the homomorphism $h: \mathbb{S} \rightarrow \operatorname{GO}(H, \phi)_{\mathbb{R}}$ that defines the Hodge structure on $H$ lifts to a homomorphism $\tilde{h}: \mathbb{S} \rightarrow \operatorname{CSpin}(H, \phi)_{\mathbb{R}}$. Cf. [26], 2.2.3-4. By contrast, the Mumford-Tate group of $H(1)$ is the special orthogonal group $\mathrm{SO}(H, \phi)$. We can still lift to $\operatorname{CSpin}(H, \phi)$, but the homomorphism $\operatorname{CSpin}(H, \phi) \rightarrow \mathrm{SO}(H, \phi)$ is not an isogeny.

6.4 As a preparation for the main result of this section, we need to recall some facts about the moduli of polarized K3 surfaces. We closely follow Rizov [17, [18].

Fix a natural number $d$. Let $\left(L_{0}, \psi\right)$ be the quadratic lattice $U^{\oplus 3} \oplus E_{8}^{\oplus 2}$ (with $U$ the hyperbolic lattice). With $\left\{e_{1}, f_{1}\right\}$ the standard basis of the first copy of $U$, let $\left(L_{2 d}, \psi_{2 d}\right)$ be the sublattice $\left\langle e_{1}+d f_{1}\right\rangle \oplus U^{\oplus 2} \oplus E_{8}^{\oplus 2}$ of $L_{0}$. In what follows we write $\mathrm{SO}$ for the $\mathbb{Z}$-group scheme $\operatorname{SO}\left(L_{2 d}, \psi_{2 d}\right)$. For $n \geq 1$, let $K(n) \subset \mathrm{SO}\left(\mathbb{A}_{f}\right)$ be the compact open subgroup of elements in $\mathrm{SO}(\hat{\mathbb{Z}})$ that reduce to the identity modulo $n$. If $K$ is an open subgroup of $K(n)$ for some $n \geq 3$, Rizov defines in [17], Section 6, a moduli stack $\mathscr{F}_{2 d, K}$ over $\mathbb{Q}$ of K3 surfaces with a primitive polarization of degree $2 d$ and a level $K$ structure. (In fact, Rizov does this over open parts of $\operatorname{Spec}(\mathbb{Z})$, but for our purposes it suffices to work over $\mathbb{Q}$.) By [18], Cor. 2.4.3, $\mathscr{F}_{2 d, K}$ is a scheme. If $(Y, \lambda)$ is a $\mathrm{K} 3$ surface over a field $k$ of characteristic 0 equipped with a primitive polarization of degree $2 d$, a level $K(n)$-structure on $(Y, \lambda)$ is an isometry $H_{\text {prim }}^{2}\left(Y_{\bar{k}}, \mathbb{Z} / n \mathbb{Z}\right)(1) \stackrel{\sim}{\rightarrow} L_{2 d} / n L_{2 d}$. (See [17], Example 5.1.3.)

The construction of 3.1 has an analogue in this setting. Let $\mathscr{F}_{0, \mathbb{C}}$ be an irreducible component of $\mathscr{F}_{2 d, K(3)} \otimes \mathbb{C}$, and let $F \subset \mathbb{C}$ be its field of definition, so that we have a geometrically irreducible component $\mathscr{F}_{0} \subset \mathscr{F}_{2 d, K(3)} \otimes F$. For $K \subset K(3)$ we have an étale morphism $\mathscr{F}_{K, K(3)}: \mathscr{F}_{2 d, K} \rightarrow \mathscr{F}_{2 d, K(3)}$, which for $K$ normal in $K(3)$ is Galois with group $K(3) / K$. Let $\mathscr{F}_{K} \subset \mathscr{F}_{2 d, K} \otimes F$ be the inverse image of $\mathscr{F}_{0}$. Suppose we are given a compatible collection $\bar{y}=\left(\bar{y}_{K}\right)$ of geometric points of the $\mathscr{F}_{K}$, for $K$ open and normal in $K(3)$. We write $\bar{y}_{0}$ for $\bar{y}_{K(3)}$. We then have and associated homomorphism

$$
\Phi_{\bar{y}}: \pi_{1}\left(\mathscr{F}_{0}, \bar{y}_{0}\right) \rightarrow K(3) \subset \mathrm{SO}(\hat{\mathbb{Z}})
$$

6.5 With $\mathrm{SO}$ as above, let $\Omega^{ \pm}$be the space of homomorphisms $h: \mathbb{S} \rightarrow \mathrm{SO}_{\mathbb{R}}$ that give $L_{2 d} \otimes \mathbb{Q}$ a Hodge structure of type $(-1,1)+(0,0)+(1,-1)$ with Hodge numbers $1-19-1$, such that $\pm \psi_{2 d}$ is a polarization. The group $\mathrm{SO}(\mathbb{R})$ acts transitively on $\Omega^{ \pm}$, and the pair $\left(\mathrm{SO}_{\mathbb{Q}}, \Omega^{ \pm}\right)$is a Shimura datum of abelian type with reflex field $\mathbb{Q}$.

On of the main results of [18] (loc. cit., Thm. 3.9.1) is that for an open subgroup $K \subset K(3)$ there 
is an étale morphism of $\mathbb{Q}$-schemes

$$
j_{K}: \mathscr{F}_{2 d, K} \rightarrow \mathrm{Sh}_{K}\left(\mathrm{SO}_{\mathbb{Q}}, \Omega^{ \pm}\right)
$$

in such a way that for $K_{2} \subset K_{1}$ the diagram

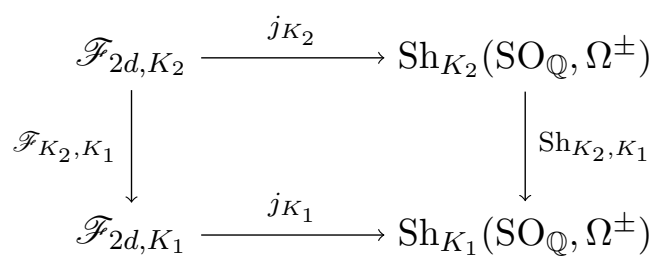

is cartesian. The image of $j_{K}$ is the complement of a divisor (ibid., 3.10(B)).

6.6 Theorem. Let $Y$ be a K3 surface over a subfield $k \subset \mathbb{C}$ that is finitely generated over $\mathbb{Q}$. Let $H=$ $H^{2}(Y(\mathbb{C}), \mathbb{Z})(1)$, and let $G_{\mathrm{B}} \subset \mathrm{GL}(H)$ be the Mumford-Tate group. Let $\rho_{Y}: \operatorname{Gal}(\bar{k} / k) \rightarrow \mathrm{GL}(H)(\hat{\mathbb{Z}})$ be the Galois representation on $\hat{H}=H^{2}\left(Y_{\bar{k}}, \hat{\mathbb{Z}}\right)(1)$, which we identify with $H \otimes \hat{\mathbb{Z}}$ via the comparison isomorphism between singular and étale cohomology. Then the image of $\rho_{Y}$ has a subgroup of finite index which is an open subgroup of $G_{\mathrm{B}}(\hat{\mathbb{Z}})$.

Proof. The proof is very similar to that of Theorem [5.3. The main difference is that for K3 surfaces the Mumford-Tate conjecture is known, due to results of Tankeev [22] and André [1], and that by Proposition 6.2 the Hodge structure on $H$ is always Hodge-maximal.

We retain the notation introduced in 6.4 and 6.5. Choose a primitive polarization $\lambda$ on $Y$, say of degree $2 d$. Further choose an isometry $i: H \stackrel{\sim}{\rightarrow} L_{2 d}$. These choices give us a compatible system $\bar{y}=\left(\bar{y}_{K}\right)$ of points $\bar{y}_{K} \in \mathscr{F}_{2 d, K}(\mathbb{C})$, where $K$ runs through the set of open subgroups of $K(3)$. Possibly after replacing $k$ with a finite extension in $\mathbb{C}$, we may assume that $k=k^{\text {conn }}$ and that $\bar{y}_{0}=\bar{y}_{K(3)}$ comes from a $k$-valued point $y_{0} \in \mathscr{F}_{2 d, K(3)}(k)$ by composing it with the embedding $k \hookrightarrow \mathbb{C}$. Of course, $y_{0}$ is just the moduli point of $(Y, \lambda)$ equipped with a suitable level 3 structure.

Via the chosen isometry $i$ the Hodge structure on $H_{\mathbb{Q}}$ defines a point $h_{0} \in \Omega^{ \pm}$. Let $\bar{t}=\left(\bar{t}_{K}\right)$ be the system of $\mathbb{C}$-valued points $\left[h_{0}, e K\right]$ of $\mathrm{Sh}_{K}\left(\mathrm{SO}, \Omega^{ \pm}\right)$, and abbreviate $\bar{t}_{K(3)}$ to $\bar{t}_{0}$. The construction of the period map (6.5.1) is such that $j_{K}\left(\bar{y}_{K}\right)=\bar{t}_{K}$ for all $K \subset K(3)$.

Let $t_{0}=j_{K(3)}\left(y_{0}\right)$, which is a $k$-valued point of $\operatorname{Sh}_{K(3)}\left(\mathrm{SO}, \Omega^{ \pm}\right)$. Let $\mathscr{F}_{0} \subset \mathscr{F}_{2 d, K(3)} \otimes k$ and $\mathscr{S}_{0} \subset \mathrm{Sh}_{K(3)}\left(\mathrm{SO}, \Omega^{ \pm}\right) \otimes k$ be the irreducible components containing $\bar{y}_{0}$ and $\bar{t}_{0}$, respectively; as they are smooth over $k$ and have a $k$-rational point, these components are geometrically irreducible. By construction, $j_{0}=j_{K(3)}$ restricts to an étale morphism $j_{0}: \mathscr{F}_{0} \rightarrow \mathscr{S}_{0}$ over $k$.

Consider the homomorphism $\phi_{\bar{t}}: \pi_{1}\left(\mathscr{S}_{0}, \bar{t}_{0}\right) \rightarrow K(3)$ as in 3.1. We also have the homomorphism $\Phi_{\bar{y}}: \pi_{1}\left(\mathscr{F}_{0}, \bar{t}_{0}\right) \rightarrow K(3)$ of (6.4.1). (In both cases we have now extended the base field to $k$.) The fact that the diagrams (6.5.2) are Cartesian implies that $\Phi_{\bar{y}}=\phi_{\bar{t}} \circ j_{0, *}$.

Let $H_{\text {prim }} \subset H$ be the primitive integral cohomology, and identify the primitive étale cohomology with $\hat{\mathbb{Z}}$-coefficients $\hat{H}_{\text {prim }} \subset \hat{H}$ with $H_{\text {prim }} \otimes \hat{\mathbb{Z}}$. Via the chosen isometry $i$, the Galois action on $\hat{H}_{\text {prim }}$ is a representation $\rho_{Y, \operatorname{prim}}: \operatorname{Gal}(\bar{k} / k) \rightarrow \mathrm{SO}(\hat{\mathbb{Z}})$. Note that the Galois action $\rho_{Y}$ on $\hat{H}$ leaves $\hat{H}_{\text {prim }}$ stable and is trivial on the complement; hence the image of $\rho_{Y}$ is the same as the image of $\rho_{Y \text {,prim. }}$. On the other hand, the $k$-rational point $y_{0}$ gives rise to a section $\sigma_{y_{0}}$ of the homomorphism $\pi_{1}\left(\mathscr{F}_{0}, \bar{y}_{0}\right) \rightarrow \operatorname{Gal}(\bar{k} / k)$ induced by the structural morphism $\mathscr{F}_{0} \rightarrow \operatorname{Spec}(k)$. The composition $\Phi_{\bar{y}} \circ \sigma_{y_{0}}: \operatorname{Gal}(\bar{k} / k) \rightarrow K(3) \subset$ $\mathrm{SO}(\hat{\mathbb{Z}})$ is the same as $\rho_{Y, \text { prim. }}$. The composition $j_{0, *} \circ \sigma_{y_{0}}: \operatorname{Gal}(\bar{k} / k) \rightarrow \pi_{1}\left(\mathscr{S}_{0}, \bar{t}_{0}\right)$ is the section $\sigma_{t_{0}}$ given by the point $t_{0} \in \mathscr{S}_{0}(k)$. It follows that $\phi_{\bar{t}} \circ \sigma_{t_{0}}: \operatorname{Gal}(\bar{k} / k) \rightarrow \mathrm{SO}(\hat{\mathbb{Z}})$ is the same as $\rho_{Y, \text { prim }}$. 
The rest is the same as in the proof of Theorem 5.3. Let $G_{\mathrm{B}} \subset \mathrm{GSp}_{2 g}$ be the Mumford-Tate group, and let $X \subset \Omega^{ \pm}$be the $G_{\mathrm{B}}(\mathbb{R})$-orbit of $h_{0}$. The pair $\left(G_{\mathrm{B}}, X\right)$ is a Shimura datum and we have a morphism $f:\left(G_{\mathrm{B}}, X\right) \rightarrow\left(\mathrm{SO}, \Omega^{ \pm}\right)$. Let $K_{0}=f^{-1}(K(3))$, let $E$ be the reflex field, and let $\mathrm{Sh}(f): \mathrm{Sh}_{K_{0}}\left(G_{\mathrm{B}}, X\right) \rightarrow \mathrm{Sh}_{K(3)}\left(\mathrm{SO}, \Omega^{ \pm}\right) \otimes E$ be the morphism induced by $f$. We have a compatible system $\bar{s}$ of points $\bar{s}_{K}=\left[h_{0}, e K\right] \in \operatorname{Sh}_{K}\left(G_{\mathrm{B}}, X\right)(\mathbb{C})$ with $\operatorname{Sh}(f)(\bar{s})=\bar{t}$. The point $\bar{s}_{0}=\bar{s}_{K_{0}}$ comes from a $k$-valued point $s_{0}$, and if $S_{0} \subset \operatorname{Sh}_{K_{0}}\left(G_{\mathrm{B}}, X\right)_{k}$ is the irreducible component in which it lies, $s_{0}$ gives a section $\sigma_{s_{0}}: \operatorname{Gal}(\bar{k} / k) \rightarrow \pi_{1}\left(S_{0}, \bar{s}_{0}\right)$. Finally, if $\phi_{\bar{s}}: \pi_{1}\left(S_{0}, \bar{s}_{0}\right) \rightarrow K_{0} \subset G_{\mathrm{B}}(\hat{\mathbb{Z}})$ is the representation (3.1.1), it follows from the functoriality explained in Remark 3.2 that $\phi_{\bar{s}} \circ \sigma_{s_{0}}$ is the same as $\rho_{Y, \text { prim }}$.

By the Mumford-Tate conjecture, $s_{0}$ is $\ell$-Galois-generic with respect to $\phi_{\bar{s}}$ for every $\ell$. By Theorem 4.3 it follows that $s_{0}$ is Galois-generic, and by Corollary 3.7(iii), the theorem follows.

\section{References}

[1] Y. André, On the Shafarevich and Tate conjectures for hyper-Kähler varieties. Math. Ann. 305 (1996), no. 2, 205-248.

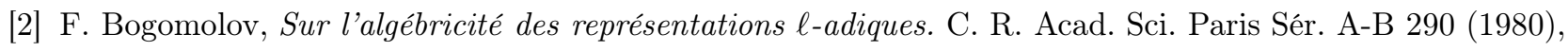
no. 15, A701-A703.

[3] M. Borovoi, Abelian Galois cohomology of reductive groups. Mem. Amer. Math. Soc. 132 (1998), no. 626.

[4] N. Bourbaki, Éléments de mathématique. Groupes et Algèbres de Lie, Chap. 4, 5 et 6. Masson, 1981.

[5] A. Cadoret, An adelic open image theorem for Abelian schemes. To appear in Int. Math. Res. Not. doi $10.1093 / \mathrm{imrn} / \mathrm{rnu} 259$

[6] A. Cadoret, A. Kret, Galois-generic points on connected Shimura varieties - I, Adelic versus $\ell$-adic. Preprint, 2015.

[7] P. Deligne, Travaux de Shimura. Sém. Bourbaki (1970/71), Exp. No. 389. In: Lecture Notes in Math. 244, Springer, Berlin, 1971; pp. 123-165.

[8] P. Deligne, Variétés de Shimura: interprétation modulaire, et techniques de construction de modèles canoniques. In: Automorphic forms, representations and L-functions (Proc. Symp. Pure Math. 33, Part 2), pp. 247-289. A.M.S., 1979.

[9] C. Hui, M. Larsen, Adelic openness without the Mumford-Tate conjecture. Preprint, arXiv:1312.3812.

[10] M. Larsen, R. Pink, On $\ell$-independence of algebraic monodromy groups in compatible systems of representations. Invent. Math. 107 (1992), no. 3, 603-636.

[11] M. Larsen, R. Pink, Abelian varieties, $\ell$-adic representations, and $\ell$-independence. Math. Ann. 302 (1995), no. $3,561-579$.

[12] D. Lombardo, On the $\ell$-adic Galois representations attached to nonsimple abelian varieties. Preprint, arXiv: 1402.1478

[13] J. Milne, K.-y. Shih, Conjugates of Shimura varieties. In: Hodge cycles, motives, and Shimura varieties. Lecture Notes in Math. 900, Springer, 1982; pp. 280-356.

[14] R. Pink, $\ell$-adic algebraic monodromy groups, cocharacters, and the Mumford-Tate conjecture. J. reine angew. Math. 495 (1998), 187-237.

[15] V. Platonov, A. Rapinchuk, Algebraic groups and number theory. Pure and Applied Mathematics 139. Academic Press, Inc., Boston, MA, 1994.

[16] H. Pohlmann, Algebraic cycles on abelian varieties of complex multiplication type. Ann. of Math. (2) 88 (1968), 161-180.

[17] J. Rizov, Moduli stacks of polarized K3 surfaces in mixed characteristic. Serdica Math. J. 32 (2006), no. 2-3, $131-178$.

[18] J. Rizov, Kuga-Satake abelian varieties of K3 surfaces in mixed characteristic. J. reine angew. Math. 648 (2010), 13-67. 


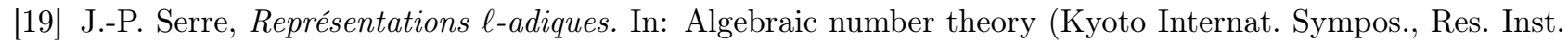
Math. Sci., Univ. Kyoto, Kyoto, 1976), pp. 177-193. Japan Soc. Promotion Sci., Tokyo, 1977. [=(Euvres 112.]

[20] J.-P. Serre, Lettre à Ken Ribet du 1/1/1981. In: CEuvres, Volume IV, number 133.

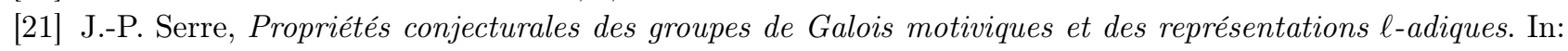
Motives (Proc. Symp. Pure Math. 55, Part 1), pp. 377-400. A.M.S., 1994. [=(Euvres 161.]

[22] S. Tankeev, Surfaces of K3 type over number fields and the Mumford-Tate conjecture. I, II. Math. USSRIzv. 37 (1991), no. 1, 191-208, and Izv. Math. 59 (1995), no. 3, 619-646.

[23] J. Tits, Classification of algebraic semisimple groups. In: Algebraic Groups and Discontinuous Subgroups (Proc. Sympos. Pure Math. 9), pp. 33-62. A.M.S., 1966.

[24] E. Ullmo, A. Yafaev, Mumford-Tate and generalised Shafarevich conjectures. Ann. Math. Qué. 37 (2013), no. 2, 255-284.

[25] B. van Geemen, Real multiplication on K3 surfaces and Kuga-Satake varieties. Michigan Math. J. 56 (2008), no. 2, 375-399.

[26] J.-P. Wintenberger, Relèvement selon une isogénie de systèmes $\ell$-adiques de représentations galoisiennes associés aux motifs. Invent. Math. 120 (1995), no. 2, 215-240.

[27] J.-P. Wintenberger, Une extension de la théorie de la multiplication complexe. J. reine angew. Math. 552 (2002), 1-14.

[28] J.-P. Wintenberger, Démonstration d'une conjecture de Lang dans des cas particuliers. J. reine angew. Math. 553 (2002), 1-16.

[29] Yu. Zarhin, Hodge groups of K3 surfaces. J. reine angew. Math. 341 (1983), 193-220.

anna.cadoret@math.polytechnique.fr

Centre de Mathématiques Laurent Schwartz - École Polytechnique, 91128 Palaiseau, France

b.moonen@science.ru.nl

Radboud University Nijmegen, IMAPP, Nijmegen, The Netherlands 